\title{
Circumnuclear star formation in Mrk 42 mapped with Gemini Near-infrared Integral Field Spectrograph
}

\author{
Moiré G. Hennig, ${ }^{\star}{ }^{\star}$ Rogemar A. Riffel, ${ }^{1 \star}$ O. L. Dors, ${ }^{2}$ Rogerio Riffel, ${ }^{3}$ \\ Thaisa Storchi-Bergmann ${ }^{3}$ and Luis Colina ${ }^{4,5}$ \\ ${ }^{1}$ Departamento de Física, CCNE, Universidade Federal de Santa Maria, 97105-900 Santa Maria, RS, Brazil \\ ${ }^{2}$ Universidade do Vale do Paraíba, Av. Shishima Hifumi 2911, CEP 12244-000 São José dos Campos, SP, Brazil \\ ${ }^{3}$ Departamento de Astronomia, IF, Universidade Federal do Rio Grande do Sul, CP 15051, $91501-970$ Porto Alegre, RS, Brazil \\ ${ }^{4}$ Centro de Astrobiología (CAB, CSIC-INTA), Carretera de Ajalvir, E-28850 Torrejón de Ardoz, Madrid, Spain \\ ${ }^{5}$ ASTRO-UAM, Universidad Autónoma de Madrid (UAM), Unidad Asociada CSIC, E-28049 Madrid, Spain
}

Accepted 2018 February 26. Received 2018 February 26; in original form 2017 July 12

\begin{abstract}
We present Gemini Near-infrared Integral Field Spectrograph (NIFS) observations of the inner $1.5 \times 1.5 \mathrm{kpc}^{2}$ of the narrow-line Seyfert 1 galaxy Mrk 42 at a spatial resolution of $60 \mathrm{pc}$ and spectral resolution of $40 \mathrm{~km} \mathrm{~s}^{-1}$. The emission-line flux and equivalent width maps clearly show a ring of circumnuclear star formation regions surrounding the nucleus with radius of $\sim 500 \mathrm{pc}$. The spectra of some of these regions show molecular absorption features which are probably of $\mathrm{CN}$, TiO, or $\mathrm{VO}$, indicating the presence of massive evolved stars in the thermally pulsing asymptotic giant branch phase. The gas kinematics of the ring is dominated by rotation in the plane of the galaxy, following the large-scale disc geometry, while at the nucleus an additional outflowing component is detected blueshifted by $300-500 \mathrm{~km} \mathrm{~s}^{-1}$, relative to the systemic velocity of the galaxy. Based on the equivalent width of $\mathrm{Br} \gamma$ we find pieces of evidence of gradients in the age of $\mathrm{H}_{\text {II }}$ regions along the ring of Mrk 42, favouring the pearls on a string scenario of star formation. The broad component of $\mathrm{Pa} \beta$ emission line presents a Full Width at Half Maximum of $\sim 1480 \mathrm{~km} \mathrm{~s}^{-1}$, implying in a mass of $\sim 2.5 \times 10^{6} \mathrm{M}_{\odot}$ for the central supermassive black hole. Based on emission-line ratios we conclude that besides the active galactic nucleus, Mrk 42 presents nuclear Starburst activity.
\end{abstract}

Key words: galaxies: active - galaxies: individual: (Mrk 42) - galaxies: ISM - infrared: galaxies.

\section{INTRODUCTION}

Since the early studies by Morgan (1958) and Sérsic \& Pastoriza (1967) - see also Kennicutt et al. (1989) - that showed that there are many spiral galaxies hosting circumnuclear star formation regions (CNSFRs), much progress has been done in our knowledge about how are these regions originated. Inflows of gas from hundred of parsec scales towards the centre of galaxies are now commonly observed (e.g. Riffel et al. 2008; Riffel, Storchi-Bergmann \& Winge 2013b; Diniz et al. 2015). These inflows, besides possibly feeding the central supermassive black hole (SMBH) can also trigger (circum-)nuclear star formation, such as it has been observed in some active galaxies. This relation between nuclear activity and star formation is commonly called as the active galactic nucleusStarburst (AGN-Starburst) connection (Perry \& Dyson 1985; Norman \& Scoville 1988). Studies on AGN-Starburst connections are of fundamental importance to explain the possible co-evolution of SMBH and its host galaxy, as the star formation in the central region leads to the growth of the bulge mass, while gas accreted by the SMBH leads to its growth (Ferrarese \& Merritt 2000; Gebhardt et al. 2000).

In barred galaxies the material probably flows through the largescale bars to reach the circum-nuclear region (Sakamoto et al. 1999; Jogee, Scoville \& Kenney 2005), where it can be accumulated and forming a gas reservoir favouring the star formation in circumnuclear rings with radius of a few hundreds of parsecs (Simões et al. 2007; Böker et al. 2008). Inflows of ionized and molecular gas within the inner kiloparsec of nearby active galaxies have also been observed in association with dust spirals using optical (e.g. Schnorr-Müller al. 2014a,b) and near-infrared (hereafter near-IR, e.g. Riffel et al. 2008, 2013a) integral field spectroscopy (IFS). Rings of circumnuclear star-formation regions (CNSFRs) are commonly observed in spiral galaxies, being associated with the inner and outer Lindblad resonances and can be produced by bar or oval distortion perturbations (e.g. Schwarz 1981). Such rings are more 
frequently found in AGN hosts in comparison to normal galaxies or Starbursts (Hunt \& Malkan 1999; Hunt et al. 1999). In addition, lowionization nuclear emission-line regions hosts show higher fractions of inner rings, while for Seyfert galaxies outer rings are more often observed, with ring fractions being several times larger than in normal galaxies (Hunt \& Malkan 1999). Regarding the presence of bars, Hunt \& Malkan (1999) found that active and non-active galaxies show similar incidence of them, thus the distinct fraction of rings seems to be the only morphological difference between active and non-active galaxies.

Several recent studies were aimed in constraining the physical properties of CNSFRs in active and non-active galaxies (Davies et al. 2007; Böker et al. 2008; Dors et al. 2008; Falcón-Barroso et al. 2014; van der Laan et al. 2015; Riffel et al. 2016). CNSFRs show star-formation rates (SFRs) of $10^{-3}$ to $10^{-1} \mathrm{M}_{\odot} \mathrm{yr}^{-1}$ and have ionized gas masses ranging from $10^{4}$ to $10^{5} \mathrm{M}_{\odot}$ (e.g. Dors et al. 2008; Riffel et al. 2016). The scenario that leads to the formation of CNFRs is still an open issue and the way the material settles in the circum-nuclear region may represent different processes for the star formation. For example, Knapen (2005) considers a distinction between CNSFRs and nuclear star formation regions: in the former, the material that arrives in the central region forms a circum-nuclear ring with $\sim 2 \mathrm{kpc}$ radius, while in the latter the material settles in more internal regions (within a few hundred parsecs). Two main scenarios have been proposed for the origin of CNSFRs: the popcorn and pearls on a string scenarios (Elmegreen 1994; Böker et al. 2008). In the first scenario, after an increase in the gas density due to accumulation of material in the ring, the stellar clusters are formed at random positions. In this scenario, there are no age sequences for the star clusters along the ring. In the second scenario, the gas flows to the central region along bars and is accumulated at two locations (at the tips of the bar), originating overdense regions - ODR, in which the stars are formed. Due to the differential rotation of the bar and the disc of the galaxy, the star formation 'moves' along the ring, leading to a sequence of ages for young stellar clusters.

Until now, studies on star formation in CNSFRs seem to have not produced yet conclusive results about the star formation scenario. In fact, Mazzuca et al. (2008), who used photometric $\mathrm{H} \alpha$ data of 22 CNSFRs, found that about half of the rings contain azimuthal age gradients, while Dors et al. (2008) and Riffel et al. (2016) did not found any sequence of age in CNSFRs located in three galaxies, using optical and near-IR IFS.

With the goal to improve the knowledge of star formation in CNSFRs, increasing the sample of objects, we have used near-IR IFS to study the circumnuclear star formation and nuclear activity in the nearby active galaxy Mrk 42. This object is classified as an SBa galaxy harbouring a Narrow-Line Seyfert 1 nucleus (Malkan et al. 1998), being located at a redshift $z=0.024634$ (Falco et al. 1999), corresponding to a distance of $102.6 \mathrm{Mpc}$ by assuming a Hubble constant $H_{0}=72 \mathrm{~km} \mathrm{~s}^{-1} \mathrm{Mpc}^{-1}$. At this distance, 1 arcsec corresponds to $497 \mathrm{pc}$ at the galaxy. It harbours an SMBH of mass $(0.7-18) \times 10^{6}$ as derived from scaling relations and based on the X-ray excess variance (Wang \& Lu 2001; Bian \& Zhao 2003; Nikolajuk, Czerny \& Gurynowicz 2009).

Muñoz Marín et al. (2007) present an atlas of the central regions of 75 Seyfert galaxies imaged in the near-UV with the Hubble Space telescope (HST), including Mrk 42 in their sample. They found that Mrk 42 has a very bright compact nucleus with a ring of star formation $\approx 300 \mathrm{pc}$ of radius, with many stellar clusters individually resolved. Indeed, the observed spiral arm seen in Mrk 42 is very coiled, being described by Storchi-Bergmann (2008) as clear ring of star formation surrounding the nucleus. The size of the ring of CNSFRs observed in Mrk 42 is similar to that derived for the rings in other galaxies, such as NGC 1068 and NGC 7469, resulting from a gas ring formed through bar-forcing at the inner Lindblad resonance (e.g. Wilson et al. 1991). A broad-band HST image of Mrk 42 obtained with the Wide Field Planetary Camera 2 (WFPC2) through the filter $F 606 \mathrm{~W}$ shows grand-design nuclear spiral arms, originating from a bar with an extent of 15 arcsec oriented along position angle $\mathrm{PA}=-20 / 160^{\circ}$, and the CNSFRs ring is clearly observed (Malkan, Gorjian \& Tam 1998; Deo, Crenshaw \& Kraemer 2006). The nuclear region of Mrk 42 presents strong $6.2 \mu \mathrm{m} \mathrm{PAH}$ emission as revealed by Spitzer mid-infrared spectroscopy, with a luminosity of $2 \times 10^{41} \mathrm{erg} \mathrm{s}^{-1}$, providing strong evidence for intense ongoing star formation in the circumnuclear region (Sani al. 2010).

We use $J, H$, and $K$-band IFS of the inner $3 \operatorname{arcsec} \times 3 \operatorname{arcsec}$ $\left(1.5 \times 1.5 \mathrm{kpc}^{2}\right)$ of Mrk 42 to map, for the first time, its near-IR emission-line flux distributions and kinematics. This paper is organized as follows. In Section 2, we describe the observations and data reduction procedures. In Section 3 we present maps for the fluxes, flux ratios, line-of-sight velocities, and velocity dispersions of the molecular and ionized gas emission lines. These maps are discussed in Section 4 and our conclusions are presented in Section 5.

\section{OBSERVATIONS AND DATA REDUCTION}

The observations of Mrk 42 were done using the Gemini Nearinfrared Integral Field Spectrograph (NIFS, McGregor et al. 2003) operating with the ALTAIR adaptive optics module on the Gemini North telescope in 2014 May under the programme GN-2014A-Q28. The $J-, H$-, and $K$-band observations were obtained following the standard Object-Sky-Object dithering sequence, with individual exposures of $520 \mathrm{~s}$ each. We obtained eight on-source exposures for each band, and thus the total integration time is $\sim 1.2 \mathrm{~h}$ per band. The NIFS has a square field of view of $3 \operatorname{arcsec} \times 3 \operatorname{arcsec}$, which was centred at the nucleus of the galaxy during the observations.

For the $J$ band, we used the filter J_G5603, resulting in a spectral range from 1.15 to $1.35 \mu \mathrm{m}$ and a spectral resolution of $\approx 1.7 \AA$, as obtained from the full width at half-maximum (FWHM) of typical Ar calibration lamp lines. The $H$-band observations were centred at $1.65 \mu \mathrm{m}$, covering a spectral range between 1.48 and $1.80 \mu \mathrm{m}$ at a spectral resolution of $\approx 2.5 \AA$ using H_G5604 filter. In $K$ band the resulted spectral range is $2.01-2.42 \mu \mathrm{m}$, centred at $2.20 \mu \mathrm{m}$ and the observations were performed using the K_G5605 filter. The resulting spectral resolution for the $K$ band is $\approx 3.2 \AA$, as obtained from FWHM of typical ArXe wavelength calibration lamp emission lines.

The data reduction followed the standard procedure of near-IR spectroscopic data treatment and was accomplished using tasks contained in the GEMINI.NIFS IRAF ${ }^{1}$ package as well as generic IRAF tasks. The data reduction procedure included trimming of the images, flat-field correction, sky subtraction, wavelength calibration, and s-distortion correction. The telluric absorptions were removed by dividing the spectra of the galaxy by the normalized spectrum of a telluric standard star of A spectral type, observed just before or after the observations of the galaxy. These standards have also been used to flux calibrate the spectra by interpolating a blackbody function to fit the spectrum of each standard star.

\footnotetext{
${ }^{1}$ IRAF is distributed by National Optical Astronomy Observatories, which are operated by the Association of Universities for Research in Astronomy, Inc., under cooperative agreement with the National Science Foundation.
} 


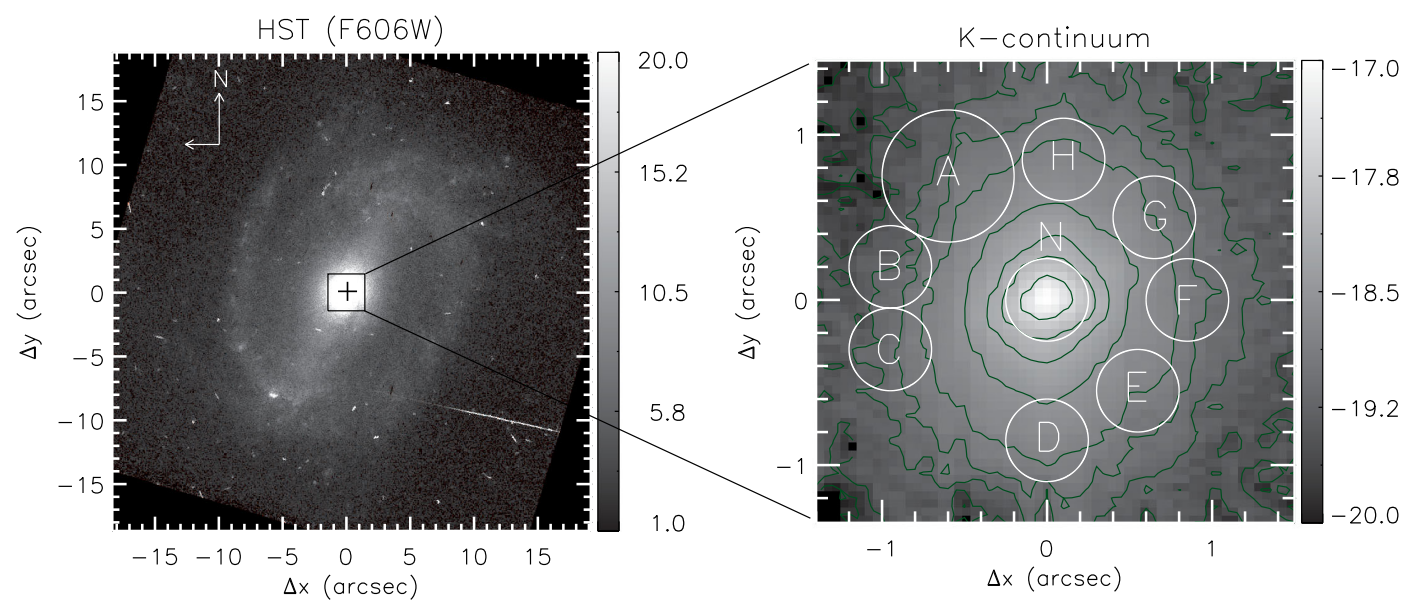

Figure 1. The left-hand panel shows an HST image of Mrk 42 obtained through the filter F606W (Malkan, Gorjian \& Tam 1998), with the FOV of the NIFS observations indicated by the central square. The right-hand panel shows the K-continuum image obtained from the NIFS data cube. The colour bars show the fluxes in arbitrary units for the HST image and in logarithmic units of $\mathrm{erg} \mathrm{cm}^{-2} \mathrm{~s}^{-1} \AA^{-1}$ for the NIFS. The circles correspond to the locations where the CNSFRs are observed, as seen in the equivalent width map for the Pa $\beta$ emission line in Fig. 4.

Finally, individual data cubes for each exposure were created with a spaxel size of $0.05 \operatorname{arcsec} \times 0.05 \operatorname{arcsec}$, which were then combined to a single data cube for each band using the gemcombine task of the GEMINI IRAF package. The final data cube for each band contains about 3600 spectra and covers the inner $3 \operatorname{arcsec} \times 3 \operatorname{arcsec}$ of the galaxy. The angular resolution is about $0.12 \mathrm{arcsec}$ for all bands, as obtained from the FWHM of the flux distribution of the telluric standard stars, corresponding to $\approx 60 \mathrm{pc}$ at the galaxy. Similar FWHM values are obtained from the flux distributions of the $\mathrm{Pa} \beta, \mathrm{Br} \gamma$, and $\mathrm{O} \mathrm{I} \lambda 1.129 \mu \mathrm{m}$ broad components.

In order to eliminate high-frequency noise from the data, but with no loss in the image quality and preserving the flux, we used the IDL script bandpass_filter.pro to apply a filter of order 3 and a cut-off frequency of $0.35 \mathrm{Ny}$ on all bands. The filter was chosen by comparing the filtered and original continuum image, making sure that there was no removal of intrinsic emission from the galaxy. Similar procedures were already applied to NGC 4303 SINFONI data cubes by our group (Riffel et al. 2016) and Butterworth filtering has extensively been used in the treatment of optical IFS of nearby galaxies (e.g. Ricci, Steiner \& Menezes 2014; May et al. 2016).

\section{RESULTS}

In the left-hand panel of Fig. 1, we present an optical image of the inner $40 \operatorname{arcsec} \times 40 \operatorname{arcsec}$ of Mrk 42 obtained with the HST WFPC2 through the filter $F 606 W$ (Malkan et al. 1998). The central cross marks the position of the nucleus indicated by ' + ' and the central square indicates the field of view (FOV) of the NIFS observations of $3 \operatorname{arcsec} \times 3$ arcsec. The nuclear grand-design spiral arms and bar are clearly seen in this image, as discussed in Deo et al. (2006). The right-hand panel of this figure shows the continuum image of the nuclear region obtained from the NIFS data cube by averaging the fluxes between 2.26 and $2.28 \mu \mathrm{m}$, a region with no strong emission/absorption lines. Although the continuum is more elongated in the direction of the bar, the presence of the bar in the inner $3 \operatorname{arcsec} \times 3 \operatorname{arcsec}$ region is not evident. In addition, the $K$-band continuum image does not show any enhancement at the locations where the CNSFRs were detected in UV continuum images (Muñoz Marín et al. 2007), which is an expected result, since young SP are enhanced in the UV spectral region while in the
near-IR just a very small fraction of their light is detected (Riffel et al. 2011). The locations of the CNSFRs are identified from the $\mathrm{Pa} \beta$ equivalent width (EqW) map shown in the bottom-left panel of Fig. 4.

In Fig. 2 we show the $J$ - (left-hand panel), $H$ - (centre panel), and $K$-band (right-hand panel) spectra for the nucleus and for the CNSFRs. These spectra were extracted within a circular aperture of 0.25 arcsec radius, centred at the nucleus and location of each CNSFR, identified as the locations of peak in the EqW map for the $\mathrm{Pa} \beta$ emission line, shown in the bottom-left panel of Fig. 4. The nucleus is labelled as ' $N$ ' and the CNSFRs from ' $A$ ' to ' $H$ ' in Fig. 4. The size of the aperture was chosen to include most of the emission form each CNSFR and for region 'A', a larger aperture with 0.4 arcsec radius was used. The spectra show several emission lines, as $\left[\mathrm{Fe}{ }_{\mathrm{II}}\right] \lambda 1.25 \mu \mathrm{m},[\mathrm{Fe} \mathrm{II}] \lambda 1.64 \mu \mathrm{m}, \mathrm{Pa} \beta, \mathrm{Br} \gamma$ and $\mathrm{H}_{2} \lambda 2.12 \mu \mathrm{m}$, as well as absorption features, as the $\mathrm{CO}$ band heads at the $K$ and $H$ bands. These spectral features can be used to investigate the origin and kinematics of the emitting gas, as well as physical properties of the stellar populations.

We fitted the profiles of the strongest emission lines by Gaussian curves in order to map their flux distributions and the gas kinematics. The fitting was done using the PROFIT (line-PROfile FITting) routine (Riffel 2010). At most locations the emission line profiles of $\mathrm{H}_{2} \lambda 2.12 \mu \mathrm{m}[\mathrm{Fe}$ II] $\lambda 1.25 \mu \mathrm{m},[\mathrm{Fe} \Pi] \lambda 1.64 \mu \mathrm{m}, \mathrm{Pa} \beta$ and $\operatorname{Br} \gamma$ are well reproduced by a single Gaussian function, but for the inner 0.45 arcsec radius the recombination lines were fitted with a narrow and a broad component, corresponding to an origin in the Narrow-Line Region and broad-line region (BLR), respectively. As the BLR is unresolved, the central wavelength and the width of the broad component were kept fixed at the values obtained by fitting the nuclear profiles shown in Fig. 2, while the amplitude of the Gaussian was allowed to vary. The line-of-sight velocity ( $\left.V_{\mathrm{LOS}}\right)$ of the broad component is blueshifted by $190 \mathrm{~km} \mathrm{~s}^{-1}$ relative to the systemic velocity of the galaxy (as derived in Section 4.3) and its FWHM is $1480 \mathrm{~km} \mathrm{~s}^{-1}$, which is larger than the values reported for $\mathrm{H} \beta$ of $670-865 \mathrm{~km} \mathrm{~s}^{-1}$ by Bian \& Zhao (2003) and Wang \& Lu (2001). Examples of fits for the $\mathrm{Pa} \beta$ and $\mathrm{H}_{2} \lambda 2.12 \mu \mathrm{m}$ profiles are shown in Fig. 3 for the nuclear position (top) and for the position of the CNSFR labelled as 'A' in Fig. 4. We used the resulting measurements for the emission-line fluxes, $V_{\mathrm{LOS}}$ and velocity dispersion 

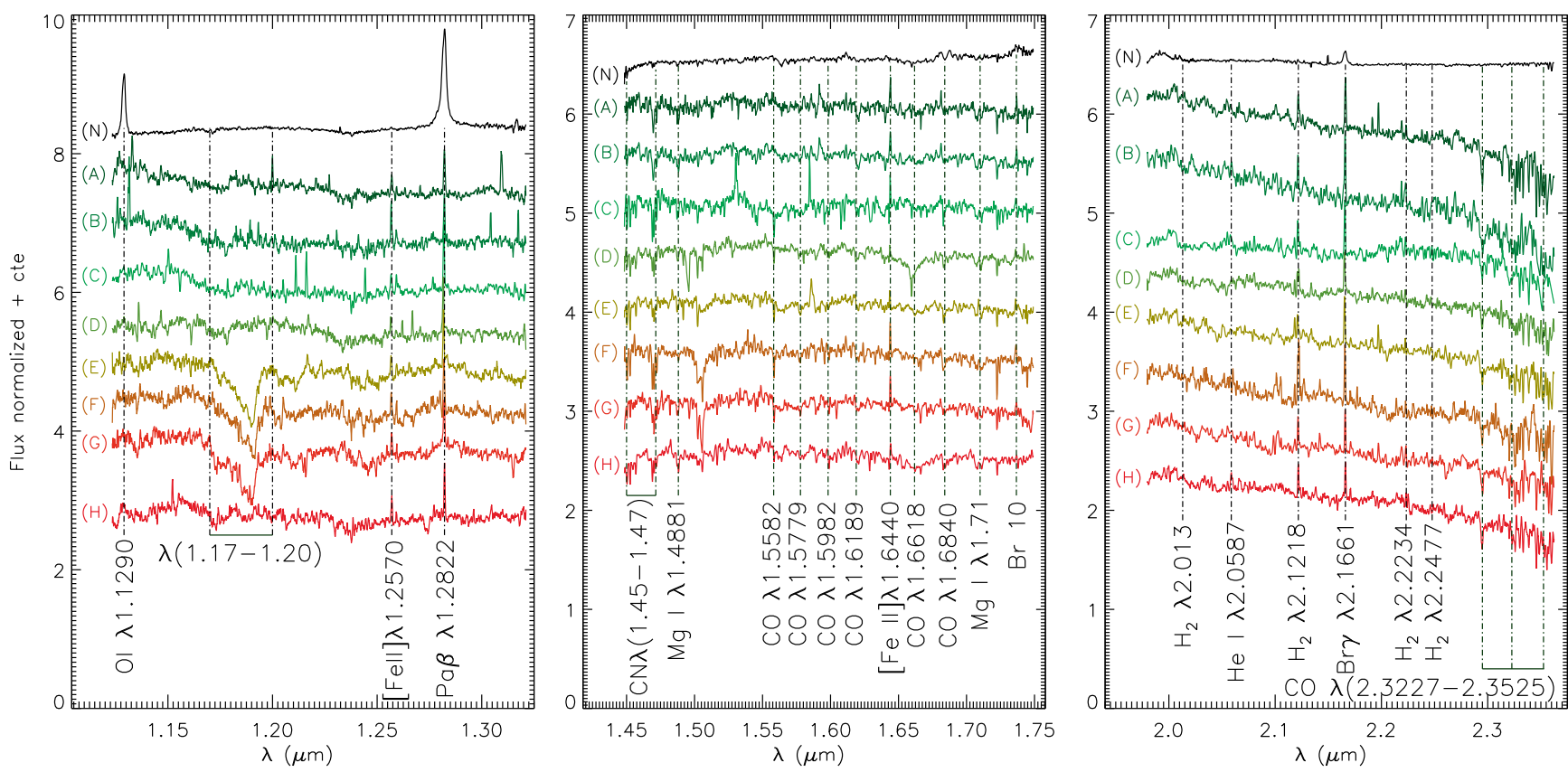

Figure 2. $J$ - (left), $H$ - (centre), and $K$-band (right) spectra for the nucleus (top spectra) and CNSFRs. The spectra were extracted for an aperture of 0.25 arcsec radius centred at the locations identified by the circles in the bottom-left panel of Fig. 4 and are corrected for the Doppler shift. The exception is the location A, for which an aperture of 0.4 arcsec radius was used. The strongest emission and absorption lines are identified for each band.
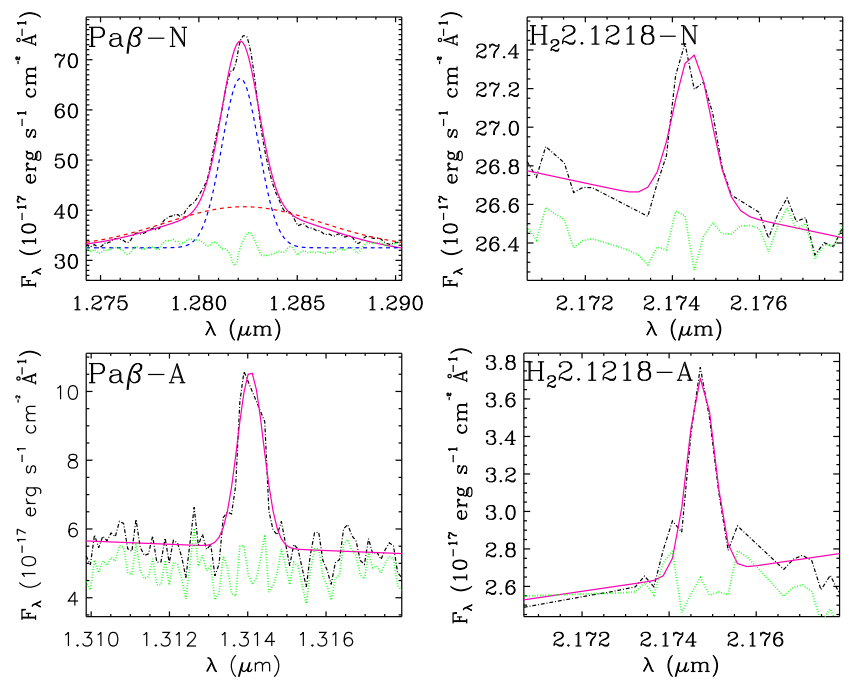

Figure 3. Examples of fits of the emission-line profiles of $\mathrm{Pa} \beta$ (left) and $\mathrm{H}_{2} \lambda 2.12 \mu \mathrm{m}$ (right) for the nucleus (top) and region A (bottom), within the apertures identified at the right-hand panel of Fig. 1. The observed profiles are shown in black, the best fitted in magenta and the residuals (plus an arbitrary constant) in green. The $\mathrm{Pa} \beta$ profile at the nucleus was fitted by two Gaussian components: the broad component is shown as a red dashed line the narrow component as a blue dashed line.

$(\sigma)$ to construct the two-dimensional maps that are presented in the following sections.

\subsection{Emission-line flux distributions and equivalent width maps}

In the top panels of Fig. 4 we present maps for the $\mathrm{Pa} \beta$ (left), [Fe $\mathrm{FI}] \lambda 1.64 \mu \mathrm{m}$ (centre), and $\mathrm{H}_{2} \lambda 2.12 \mu \mathrm{m}$ (right) emission line flux distributions. We do not show maps for [Fe $\mathrm{II}] \lambda 1.25 \mu \mathrm{m}$ and $\mathrm{Br} \gamma$ as they are very similar to those of $[\mathrm{Fe} \mathrm{II}] \lambda 1.64 \mu \mathrm{m}$ and $\mathrm{Pa} \beta$, respectively, but noisier. The grey regions in the flux maps represent masked locations where the uncertainties in flux measurements are larger than 40 percent or due to the non-detection of the emission line. The blue circle overlaid to the $\mathrm{Pa} \beta$ flux map delineates the region where two-Gaussian components were fitted to the $\mathrm{Pa} \beta$ line profile at the nuclear region. All maps clearly show the presence of the ring of CNSFRs with radius of 0.6 arcsec $(\sim 300 \mathrm{pc})$, co-spatial with the ring seen in the UV HST image (Muñoz Marín et al. 2007), but some differences are seen among the maps. While strongest extra-nuclear $\mathrm{Pa} \beta$ emission is observed for region ' $A$ ' at the north-east of the nucleus, the $\mathrm{H}_{2}$ and [ $\mathrm{Fe}$ II] emission show stronger emission from regions located at the northwest of the nucleus. In addition, $\mathrm{Pa} \beta$ show strong nuclear emission, while a weaker nuclear emission is observed in $\mathrm{H}_{2}$ and [ $\left.\mathrm{Fe} \mathrm{II}\right]$ is not detected at the nucleus of Mrk 42. In Table 1 we present flux measurements for the $[\mathrm{Fe} I \mathrm{I}] \lambda 1.25 \mu \mathrm{m}, \mathrm{Pa} \beta,[\mathrm{Fe}$ II] $\lambda 1.64 \mu \mathrm{m}$, $\mathrm{H}_{2} \lambda 2.12 \mu \mathrm{m}$ and $\mathrm{Br} \gamma$ emission lines for the nucleus and the CNSFRs integrated within a circular aperture of 0.25 arcsec radius, except for region 'A', for which an aperture of $0.4 \operatorname{arcsec}$ was used.

The bottom panels of Fig. 4 show the EqW maps for the $\mathrm{Pa} \beta$ (left), $[\mathrm{Fe}$ II $] \lambda 1.64 \mu \mathrm{m}$ (centre) and $\mathrm{H}_{2} \lambda 2.12 \mu \mathrm{m}$ (right), which can be used to better identify the CNSFRs, as the EqW measures the flux relative to the continuum emission and thus is 'a pure' line emission diagnostic. The white circles overlaid to the $\operatorname{Pa} \beta$ map (that clearly delineates the CNSFRs ring) marks the locations of the CNSFRs labelled from ' $\mathrm{A}$ ' to ' $\mathrm{H}$ ', as well as the position of the nucleus ('N'). The size of the aperture was chosen to be larger than our spatial resolution bin and includes most of the line emission from the CNSFRs. The grey continuous line represents the orientation of the bar seen in the optical HST image, shown in Fig. 1. The [Fe II] $\lambda 1.64 \mu \mathrm{m}$ and $\mathrm{H}_{2} \lambda 2.12 \mu \mathrm{m}$ show EqW values ranging from 

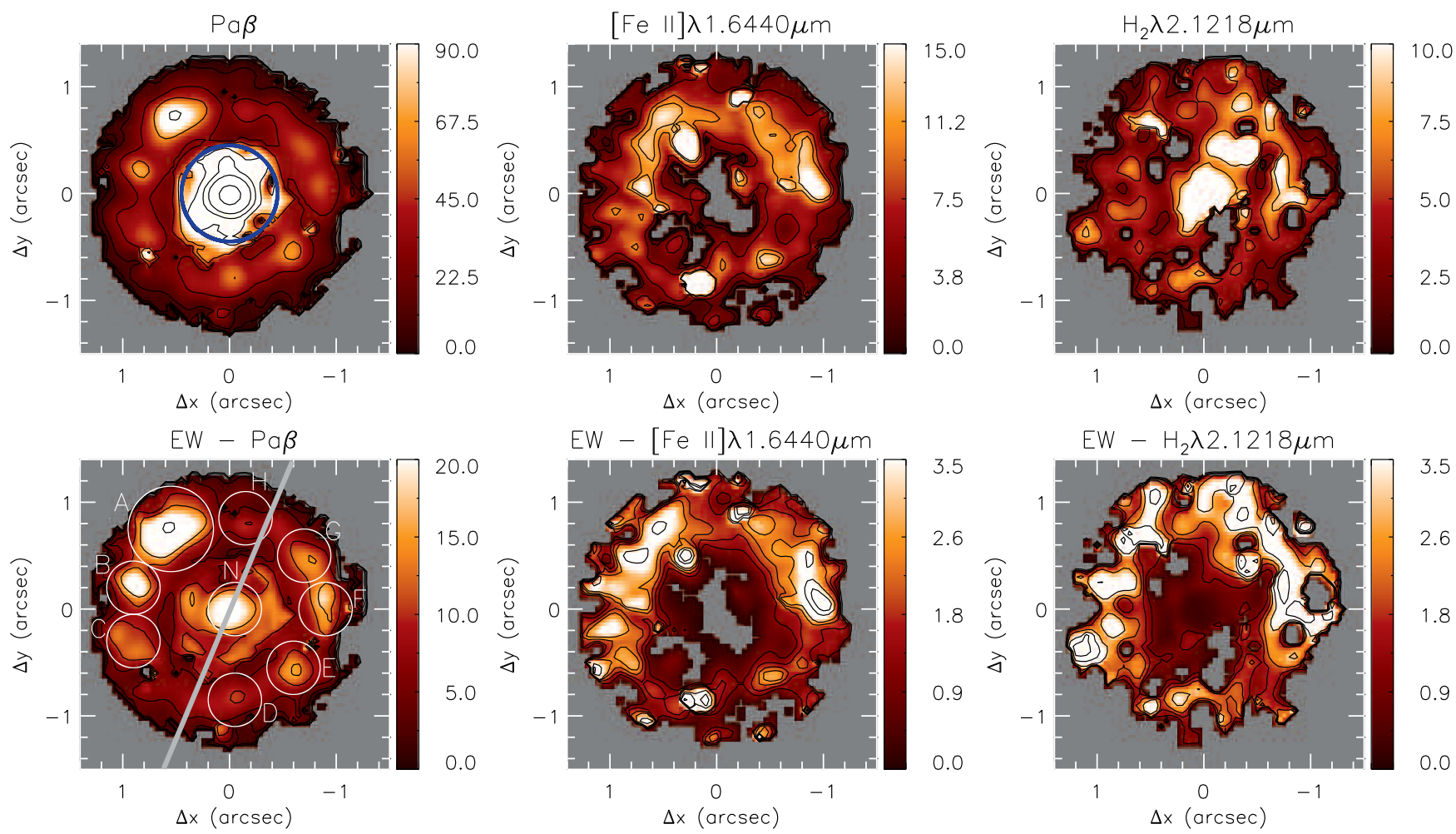

Figure 4. Top: Emission-line flux distributions for the $\mathrm{Pa} \beta$ (left), [Fe II] $\lambda 1.64 \mu \mathrm{m}$, and $\mathrm{H}_{2} \lambda 2.12 \mu \mathrm{m}$. The colour bars show the fluxes in units of $10^{-19} \mathrm{erg} \mathrm{cm}^{-2} \mathrm{~s}^{-1}$. For $\mathrm{Pa} \beta$ emission line, the blue circle around of the nucleus delineates the region $(0.45$ arcsec radius $)$ where the Pa $\beta$ profile was fitted by two Gaussian curves. Bottom: EqW maps for $\mathrm{Pa} \beta$, [Fe II] $\lambda 1.25 \mu \mathrm{m}$, and $\mathrm{H}_{2} \lambda 2.12 \mu \mathrm{m}$ emission-lines. The colour bars show the EqW in $\AA$. The circles shown in the $\mathrm{Pa} \beta$ EW map correspond to the locations of the CNSFRs and the grey line marks the orientation of the bar, as seen from the HST image of Fig. 1 .

nearly zero to $\sim 5$, while $\mathrm{Pa} \beta$ shows higher values of up to 14 . The highest values of $\mathrm{Pa} \beta \mathrm{EqW}$ are seen for regions 'A' and 'B' to the north-east of the nucleus, while the other lines show the highest values both to the north-east and north-west of the nucleus. In addition, the smallest values of EqW for the $\mathrm{H}_{2}$ are seen at the nucleus, which may be due to an increase of the continuum emission due to hot dust, as suggested by the nuclear spectrum (see Fig. 2), which is redder than the extra-nuclear spectra.

It should be noticed that the EqW values observed for all emission lines are small and a possible explanation for this is that the continuum contains an important contribution from bulge stars, that dominates the near-IR continuum emission at the central region of galaxies. In order to evaluate the contribution of the stellar bulge continuum, we followed the methodology of Dors et al. (2008) and performed aperture photometry on the continuum images to measure the continuum emission at the locations of the CNSFRs and in the surrounding regions. We concluded that the bulge contributes with about 80,85 and 90 percent of the total flux in the $K, H$, and $J$ bands, respectively. This translates to an increase in EqW values - after subtracting the contribution from the continuum - by a factor of 4 for $\mathrm{Br} \gamma$ and $\mathrm{H}_{2} \lambda 2.12 \mu \mathrm{m}$ lines, a factor 6.7 for the $[\mathrm{Fe} I] \lambda 1.64 \mu \mathrm{m}$, and a factor of 10 for the $\mathrm{Pa} \beta$ and [Fe II] $\lambda 1.25 \mu \mathrm{m}$ lines. The EqW values of $\mathrm{Pa} \beta$ and $\mathrm{Br} \gamma$ emission lines can be even larger if stars formed in previous episodes of star formation in the ring are present, but evaluating the contribution of these stars requires high signal-to-noise spectra to allow a proper spectral synthesis and this is beyond the scope of this paper.

\subsection{Emission-line ratios}

The excitation mechanisms of the $\left[\mathrm{Fe}_{\mathrm{II}}\right]$ and $\mathrm{H}_{2}$ emission lines can be investigated using the $\left[\mathrm{Fe} \mathrm{II}_{1}\right] \lambda 1.25 \mu \mathrm{m} / \mathrm{Pa} \beta$ and $\mathrm{H}_{2} / \mathrm{Br} \gamma$ line ratios, respectively (Larkin et al. 1998; Reunanen, Kotilainen \& Prieto 2002; Rodríguez-Ardila et al. 2004; Rodríguez-Ardila, Riffel \& Pastoriza 2005; Riffel et al. 2013a; Colina et al. 2015; Lamperti et al. 2017). We show maps of these line ratios for Mrk 42 in Fig. 5. The left-hand panel shows the $[\mathrm{Fe}$ II] $\lambda 1.25 \mu \mathrm{m} / \mathrm{Pa} \beta$ ratio map, while the $\mathrm{H}_{2} / \mathrm{Br} \gamma$ map is shown in the middle panel. Grey regions represent masked locations where the signal-to-noise ratio of one or both lines was not high enough to allow good fits. In addition, we do not show the values for the inner $0.45 \operatorname{arcsec}$, for which no $[\mathrm{Fe} \mathrm{II}] \lambda 1.25 \mu \mathrm{m}$ emission is detected (see Fig. 4) and the $\mathrm{H}_{2}$ and $\mathrm{Br} \gamma$ are marginally detected and thus the uncertainty in the $\mathrm{H}_{2} / \mathrm{Br} \gamma$ is high. In addition, the origin of the nuclear emission will be discussed in Section 3.2.

Both [Fe II] $\lambda 1.25 \mu \mathrm{m} / \mathrm{Pa} \beta$ and $\mathrm{H}_{2} \lambda 2.12 \mu \mathrm{m} / \mathrm{Br} \gamma$ flux ratio maps show values smaller than 0.6 at most locations, suggesting that the $\mathrm{H}_{2}$ and [Fe II] lines are excited by the star-forming regions (e.g. Riffel et al. 2013a). However, some differences are found between these maps. While $[\mathrm{Fe}$ II $] \lambda 1.25 \mu \mathrm{m} / \mathrm{Pa} \beta$ shows low values along the whole ring of CNSFRs, the $\mathrm{H}_{2} \lambda 2.12 \mu \mathrm{m} / \mathrm{Br} \gamma$ shows higher values (of up to 0.7 ) for the CNSFRs located to the north-west of the nucleus. In Table 2 we present the $[\mathrm{Fe} I I] \lambda 1.25 \mu \mathrm{m} / \mathrm{Pa} \beta$ and $\mathrm{H}_{2} \lambda 2.12 \mu \mathrm{m} / \mathrm{Br} \gamma$ line ratio values for the CNSFRs of Mrk 42, obtained by measuring the fluxes of the emission line from the integrated spectra shown in Fig. 2, and identified in Fig. 4. Small 
Table 1. Measured emission line fluxes in units of $10^{-18} \mathrm{erg} \mathrm{cm}^{-2} \mathrm{~s}^{-1}$ and the $E(B-V)$ values for the positions labelled in Fig. 4, within a circular aperture with radius showed in the second column.

\begin{tabular}{|c|c|c|c|c|c|c|c|}
\hline Region & $R(\operatorname{arcsec})$ & {$[\mathrm{Fe}$ II] $] \lambda 1.25$} & $\mathrm{~Pa} \beta$ & $\begin{array}{c}\text { Fluxes } \\
{[\mathrm{Fe} \text { II] } \lambda 1.64}\end{array}$ & $\mathrm{H}_{2} \lambda 2.12$ & $\operatorname{Br} \gamma$ & $E(B-V)[\mathrm{Pa} \beta / \mathrm{Br} \gamma]$ \\
\hline $\mathrm{N}$ & 0.25 & $105.7 \pm 59.2$ & $6420.0 \pm 190.0$ & $51.4 \pm 25.1$ & $112.7 \pm 20.0$ & $1130.0 \pm 85.0$ & $0.06 \pm 0.21$ \\
\hline B & 0.25 & $123.1 \pm 10.1$ & $258.7 \pm 21.0$ & $50.1 \pm 12.7$ & $40.5 \pm 9.7$ & $66.0 \pm 10.7$ & $0.82 \pm 0.41$ \\
\hline $\mathrm{C}$ & 0.25 & $46.8 \pm 18.5$ & $259.3 \pm 12.9$ & $75.0 \pm 18.4$ & $40.5 \pm 9.1$ & $65.7 \pm 10.4$ & $0.81 \pm 0.37$ \\
\hline $\mathrm{D}$ & 0.25 & $59.3 \pm 22.5$ & $266.2 \pm 14.6$ & $51.6 \pm 15.3$ & $39.5 \pm 8.1$ & $67.4 \pm 12.7$ & $0.81 \pm 0.45$ \\
\hline G & 0.25 & $60.1 \pm 23.9$ & $282.3 \pm 18.1$ & $83.7 \pm 18.9$ & $44.2 \pm 7.3$ & $61.3 \pm 7.9$ & $0.49 \pm 0.32$ \\
\hline $\mathrm{H}$ & 0.25 & $79.1 \pm 33.8$ & $193.9 \pm 13.8$ & $50.8 \pm 14.4$ & $50.8 \pm 7.1$ & $56.9 \pm 11.3$ & $1.11 \pm 0.49$ \\
\hline
\end{tabular}
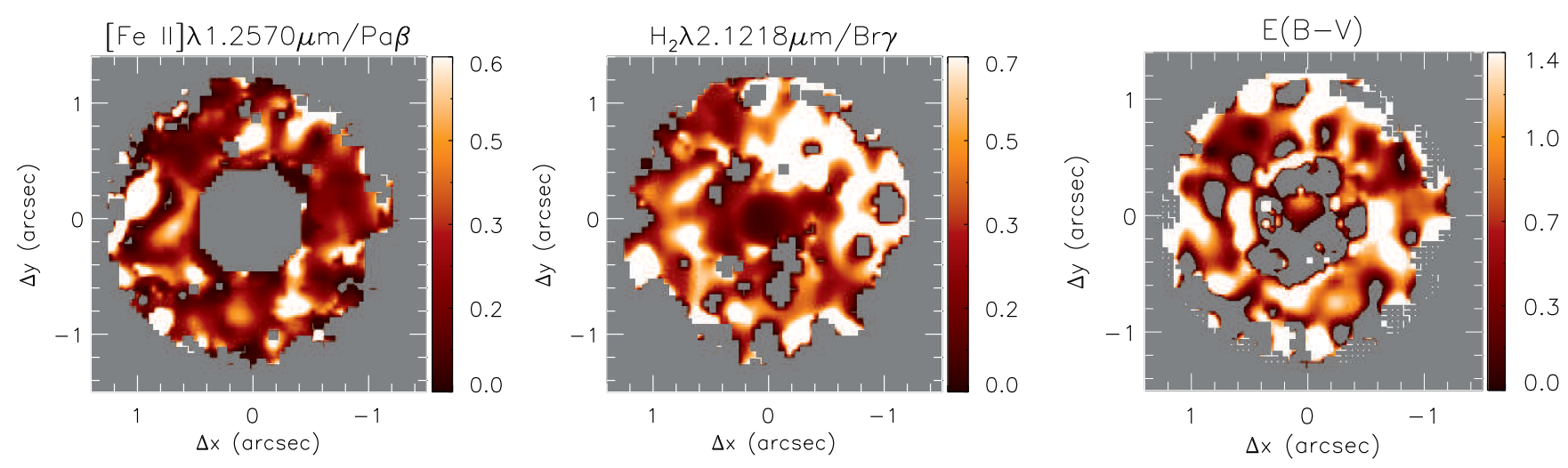

Figure 5. $[\mathrm{Fe}$ II $] \lambda 1.25 \mu \mathrm{m} / \mathrm{Pa} \beta$ (left-hand panel), $\mathrm{H}_{2} \lambda 2.12 \mu \mathrm{m} /$ Brgamma (middle panel) emission-line ratio maps and reddening map obtained from the $\mathrm{Pa} \beta /$ Brgamma ratio (right-hand panel). The grey regions represent locations where one or both lines do not present good flux measurements.

Table 2. Line ratios, velocity dispersion, and EqWs for the CNSFRs, as obtained from the integrated spectra of Fig. 2. The values for the velocity dispersion were corrected for the instrumental broadening.

\begin{tabular}{|c|c|c|c|c|c|c|c|c|c|}
\hline \multirow[b]{2}{*}{$\mathrm{P}$} & \multicolumn{2}{|c|}{ Ratios } & \multicolumn{3}{|c|}{ Velocity dispersion } & \multicolumn{4}{|c|}{ Equivalent widths } \\
\hline & {$\left[\mathrm{Fe} \mathrm{II}^{\mathrm{I}}\right] 1.25 / \mathrm{Pa} \beta$} & $\mathrm{H}_{2} 2.12 / \mathrm{Br} \gamma$ & $\begin{array}{c}\operatorname{Pa} \beta \\
\left(\mathrm{km} \mathrm{s}^{-1}\right)\end{array}$ & $\begin{array}{c}{[\mathrm{Fe} \text { II] } 1.64} \\
\left(\mathrm{km} \mathrm{s}^{-1}\right)\end{array}$ & $\begin{array}{c}\mathrm{H}_{2} 2.12 \\
\left(\mathrm{~km} \mathrm{~s}^{-1}\right)\end{array}$ & $\begin{array}{c}\mathrm{Pa} \beta \\
(\AA)\end{array}$ & $\begin{array}{c}{[\mathrm{Fe} \text { II] } 1.64} \\
(\AA)\end{array}$ & $\begin{array}{c}\mathrm{H}_{2} 2.12 \\
(\AA)\end{array}$ & $\begin{array}{l}\mathrm{Br} \gamma \\
(\AA)\end{array}$ \\
\hline B & $0.47 \pm 0.05$ & $0.61 \pm 0.18$ & $29.8 \pm 2.8$ & $36.4 \pm 11.3$ & $40.5 \pm 11.3$ & $11.4 \pm 4.1$ & $2.4 \pm 0.7$ & $3.8 \pm 1.1$ & $6.6 \pm 1.7$ \\
\hline $\mathrm{C}$ & $0.18 \pm 0.07$ & $0.62 \pm 0.17$ & $26.8 \pm 1.7$ & $50.5 \pm 14.4$ & $40.5 \pm 10.6$ & $10.0 \pm 2.7$ & $3.0 \pm 0.8$ & $3.8 \pm 1.0$ & $6.6 \pm 1.7$ \\
\hline $\mathrm{D}$ & $0.22 \pm 0.08$ & $0.59 \pm 0.16$ & $21.4 \pm 1.7$ & $35.3 \pm 13.0$ & $35.2 \pm 8.9$ & $6.8 \pm 1.8$ & $1.2 \pm 0.4$ & $2.2 \pm 0.5$ & $3.6 \pm 0.7$ \\
\hline G & $0.21 \pm 0.09$ & $0.72 \pm 0.15$ & $29.7 \pm 2.3$ & $40.5 \pm 11.1$ & $25.8 \pm 5.8$ & $9.3 \pm 2.5$ & $2.6 \pm 0.7$ & $3.0 \pm 0.6$ & $4.3 \pm 0.8$ \\
\hline $\mathrm{H}$ & $0.41 \pm 0.18$ & $0.89 \pm 0.22$ & $26.8 \pm 2.5$ & $34.3 \pm 12.2$ & $37.0 \pm 6.2$ & $5.8 \pm 2.0$ & $1.4 \pm 0.4$ & $3.1 \pm 0.5$ & $3.5 \pm 0.8$ \\
\hline
\end{tabular}

values of $\left[\mathrm{Fe}\right.$ II] $\lambda 1.25 \mu \mathrm{m} / \mathrm{Pa} \beta(<0.5)$ and $\mathrm{H}_{2} \lambda 2.12 \mu \mathrm{m} / \mathrm{Br} \gamma(<1.0)$ are observed for all CNSFRs.

The $\mathrm{Pa} \beta / \mathrm{Br} \gamma$ flux ratio can be used to map the gas extinction. The reddening $[E(B-V)]$ can be obtained by

$E(B-V)=4.74 \log \left(\frac{5.86}{F_{\operatorname{Pa} \beta} / F_{\mathrm{Br} \gamma}}\right)$,

where $F_{\mathrm{Pa} \beta}$ and $F_{\mathrm{Br} \gamma}$ are the fluxes of $\operatorname{Pa} \beta$ and $\operatorname{Br} \gamma$ emission lines, respectively. We adopted the intrinsic ratio $F_{\mathrm{Pa} \beta} / F_{\mathrm{Br} \gamma}=5.86$ corresponding to case $\mathrm{B}$ recombination for an electron temperature $T_{\mathrm{e}}=10^{4} \mathrm{~K}$ and electron density $N_{\mathrm{e}}=100 \mathrm{~cm}^{-3}$ (Osterbrock \& Ferland 2006) and used the extinction law of Cardelli, Clayton $\&$ Mathis (1989). The resulting $E(B-V)$ map is shown in the right-hand panel of Fig. 5, where values ranging from 0 to 1.2 are derived. The last column of Table 1 shows the resulting $E(B-V)$ for the nucleus and for each CNSFR. The reddening derived for the CNSFRs of Mrk 42 are consistent with those obtained for starforming regions in other galaxies and $\mathrm{H}$ II galaxies (Kotilainen et al. 2000; Martins et al. 2013a; Riffel et al. 2016).

\subsection{Gas velocity fields and velocity dispersion maps}

Gas velocity fields (top panels) and velocity dispersion (bottom panels) maps are shown in Fig. 7. Grey regions in the maps correspond to masked locations, using the same criteria as for the emissionline flux maps (see Section 3 ). The velocity fields for $\mathrm{Pa} \beta$ (top-left panel), [Fe II] (top-centre panel) and $\mathrm{H}_{2}$ (top-right panel) are presented after the subtraction of the systemic velocity of the galaxy $\left(V_{\mathrm{s}}=7390 \mathrm{~km} \mathrm{~s}^{-1}\right)$, as derived by the modelling of the $\mathrm{Pa} \beta$ velocity field by a rotating disc (see Section 4.3). At the inner 0.45 arcsec, 

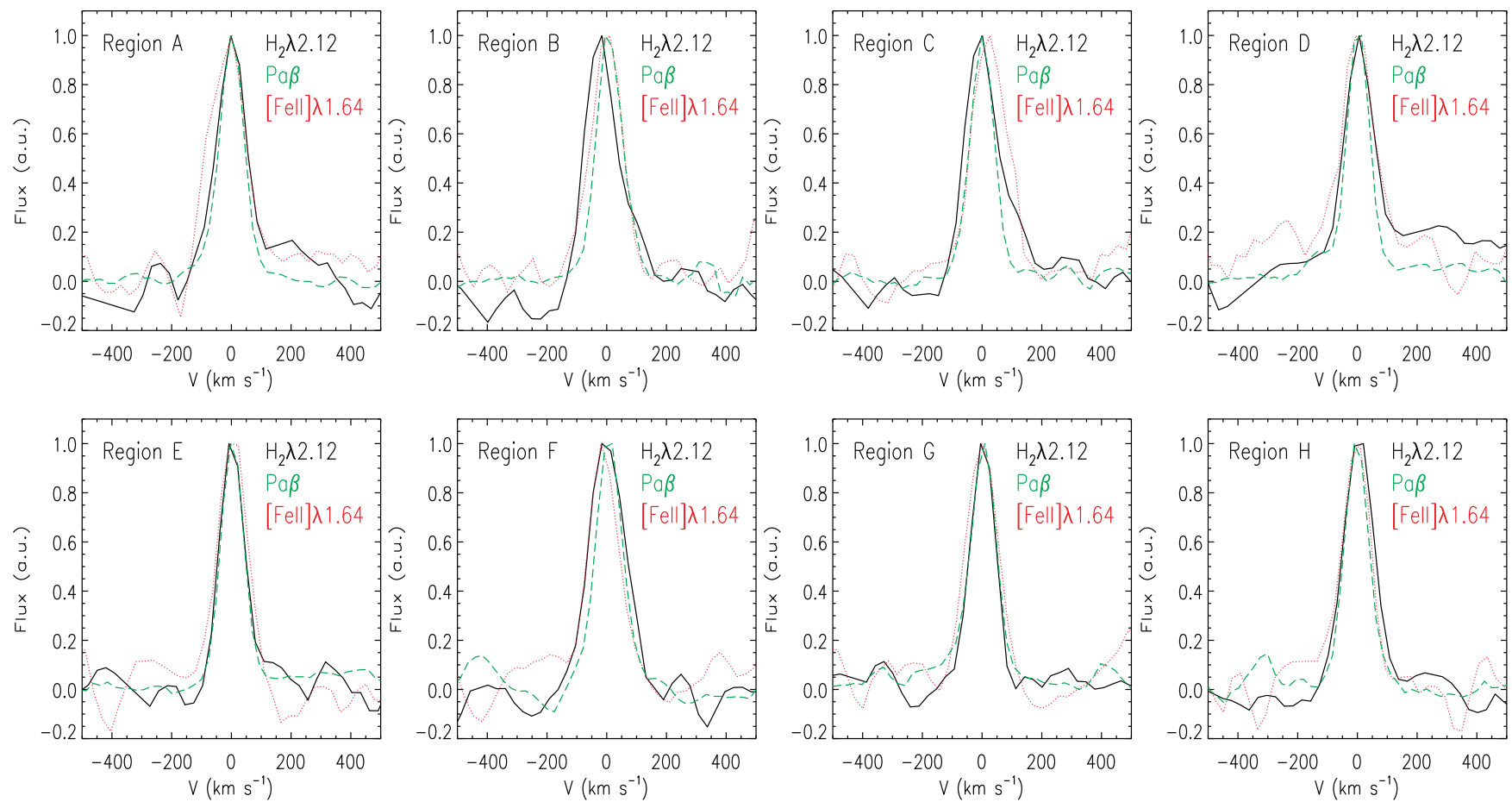

Figure 6. Emission-line profiles of $\mathrm{H}_{2} \lambda 2.12 \mu \mathrm{m}$ (black), $\mathrm{Pa} \beta$ (green), and [Fe II] $\lambda 1.64 \mu \mathrm{m}$ (red) for the CNSFRs. The panels show the normalized flux versus velocity in units of $\mathrm{km} \mathrm{s}^{-1}$ for each CNSFR.

where the $\mathrm{Pa} \beta$ profile was fitted by two Gaussian curves, we use the results for the narrow component. The velocity fields for all lines are similar and suggest gas rotation in the plane of the galaxy with the line of nodes oriented approximately along the north-south direction and a line-of-sight velocity $\left(V_{\mathrm{LOS}}\right)$ amplitude of $150 \mathrm{~km} \mathrm{~s}^{-1}$. Besides ordered rotation, in the inner 0.45 arcsec an additional velocity component is observed as indicated by the larger redshifts seen in $\mathrm{Pa} \beta$ and $\mathrm{H}_{2} V_{\text {LOS }}$ maps, possibly due to outflows from the nucleus.

The bottom panels of Fig. 7 show the velocity dispersion maps for the $\mathrm{Pa} \beta$ (left-hand panel), [Fe $\mathrm{II}$ (centre panel), and $\mathrm{H}_{2}$ (right-hand panel) emitting gas. These maps were corrected for the instrumental broadening, as $\sigma=\sqrt{\sigma_{\mathrm{obs}}^{2}-\sigma_{\text {ins }}^{2}}$, where $\sigma_{\mathrm{obs}}$ is the observed velocity dispersion and $\sigma_{\text {ins }}$ is the instrumental velocity dispersion given by $\sigma_{\text {inst }}=$ FWHM/2.355 with the FWHM measured from typical lines present on the wavelength calibration lamp (as presented in Section 2). Small values of $\sigma\left(<50 \mathrm{~km} \mathrm{~s}^{-1}\right)$ are observed along the ring of CNSFRs for all emission lines, while higher values are seen within the inner $0.45 \operatorname{arcsec}$ for the $\mathrm{Pa} \beta$ emission with $\sigma$ values of up to $200 \mathrm{~km} \mathrm{~s}^{-1}$. For illustration purpose, the $\sigma$ map for $\mathrm{Pa} \beta$ shows only values smaller than $80 \mathrm{~km} \mathrm{~s}^{-1}$, which allows the comparison with the $\mathrm{H}_{2}$ and [Fe II] $\sigma$ maps. The high $\sigma$ values seen at the nuclear region for $\mathrm{Pa} \beta$ support the interpretation that an outflow is present within the inner 0.5 arcsec. Some higher $\sigma$ values are also seen for the $\mathrm{H}_{2}$ at the nucleus, reaching values of up to $80 \mathrm{~km} \mathrm{~s}^{-1}$.

In Table 2 we present the velocity dispersion values obtained for each CNSFR by fitting the line profiles seen in the integrated spectra of Fig. 2, which suggest that the $\mathrm{Pa} \beta$ profile is usually narrower than the $\mathrm{H}_{2}$ and [Fe II] profiles. In Fig. 6 we overplot the $\mathrm{Pa} \beta,\left[\mathrm{Fe}_{\mathrm{II}}\right]$, and $\mathrm{H}_{2}$ line profiles from each CNSFR, which suggests that indeed the $\mathrm{H}_{2}$ and [Fe II] profiles are broader than that of $\mathrm{Pa} \beta$ for most regions, although it should be noticed that the $\mathrm{Pa} \beta$ is only marginally resolved in our spectra.

\section{DISCUSSION}

\subsection{Origin of the $\mathrm{H}_{2}$ and [Fe II] emission from the ring of CNSFRs}

The $[\mathrm{Fe}$ II $] \lambda 1.25 \mu \mathrm{m} / \mathrm{Pa} \beta$ line ratio can be used to investigate the excitation mechanism of the $[\mathrm{Fe} \mathrm{II}]$ emission. Some studies indicate that the $[\mathrm{Fe} I I]$ emission in active galaxies can be produced by shocks due to the interaction of radio jets with the interstellar medium (Forbes \& Ward 1993; Dopita \& Sutherland 1996), while other studies indicate that photoionization can produce most of the observed [Fe II] emission (Simpson et al. 1996; Dors et al. 2012). A way to disentangle what are the main excitation mechanisms of the [Fe II] emission is based on the use of [Fe II] $\lambda 1.25 \mu \mathrm{m} / \mathrm{Pa} \beta$ flux ratio (Reunanen et al. 2002; Rodríguez-Ardila et al. 2004, 2005; Riffel et al. 2013a; Colina et al. 2015). Typical values for Seyfert nuclei are $0.6<\left[\mathrm{Fe}_{\mathrm{II}}\right] 1.2570 \mu \mathrm{m} / \mathrm{Pa} \beta<2.0$, while smaller values than 0.6 are observed for Starburst galaxies and $\mathrm{H}$ II regions and values higher than 2.0 may be associated with shocks, e.g. due to radio jets or supernovae explosions (e.g. Rodríguez-Ardila et al. 2004). As observed in the left-hand panel of Fig. 5, the $[\mathrm{Fe}$ II $] 1.2570 \mu \mathrm{m} / \mathrm{Pa} \beta$ flux ratio map presents values smaller than 2.0 at all locations of the ring of CNSFRs, indicating that the origin of the [Fe II] line emission is photoionization by young stars.

The origin of the $\mathrm{H}_{2}$ emission in active galaxies has been addressed by several studies (e.g. Reunanen, Kotilainen \& Prieto 2002; Rodríguez-Ardila et al. 2004; Davies et al. 2005; Rodríguez-Ardila, Riffel \& Pastoriza 2005; Riffel et al. 2006; Ramos Almeida, Pérez García \& Acosta-Pulido 2009; Riffel, Storchi-Bergmann \& Nagar 2010; Dors et al. 2012; Riffel et al. 2013a; Colina et al. 2015). In summary, the $\mathrm{H}_{2}$ lines can be originated by two mechanisms: (i) fluorescent excitation through absorption of soft-UV photons, e.g. from H II regions or AGNs (Black \& van Dishoeck 1987) and (ii) collisional excitation due to heating of the gas by shocks 

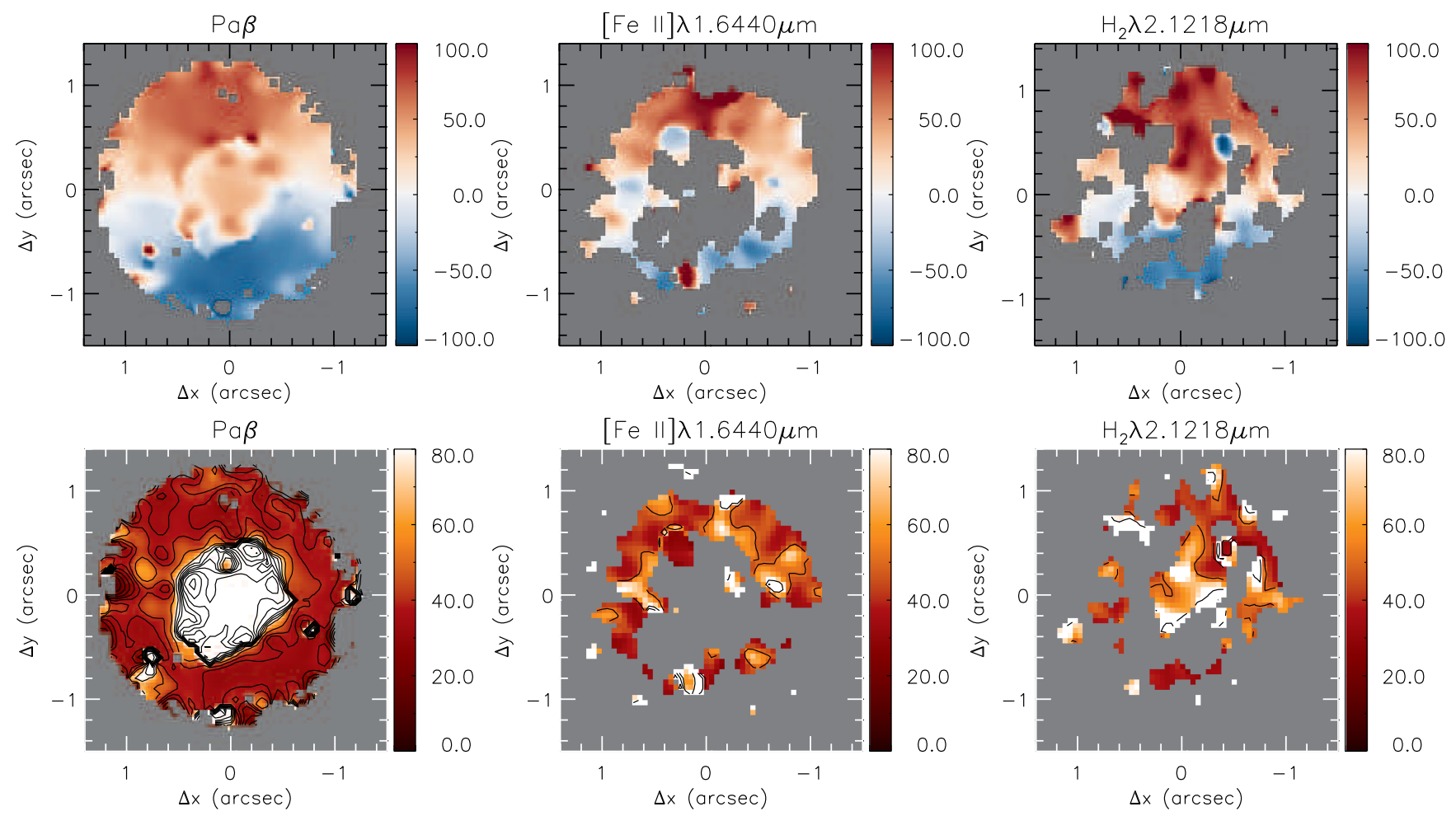

Figure 7. Gas velocity fields (top) and velocity dispersion $\sigma$ maps (bottom) for the $\mathrm{Pa} \beta$ (left), [Fe II] $\lambda 1.64 \mu \mathrm{m}$ (centre), and $\mathrm{H}_{2} \lambda 2.12 \mu \mathrm{m}$ (right) emission lines. The colour bars are shown in units of $\mathrm{km} \mathrm{s}^{-1}$.

(Hollenbach \& McKee 1989) or by X-rays from the AGN (Maloney, Hollenbach \& Tielens 1996). The $\mathrm{H}_{2} \lambda 2.12 \mu \mathrm{m} / \mathrm{Br} \gamma$ line ratio can be used to investigate the $\mathrm{H}_{2}$ main excitation mechanism: values smaller than 0.6 are commonly observed in $\mathrm{H}$ II regions and Starburst galaxies, where the $\mathrm{H}_{2}$ lines are originated by fluorescence, while larger values are observed for AGNs and supernova remnants, where heating of the gas by X-rays and shocks may dominate (e.g. Rodríguez-Ardila et al. 2005; Dors et al. 2012; Riffel et al. 2013a; Colina et al. 2015). For Mrk 42, the $\mathrm{H}_{2} \lambda 2.12 \mu \mathrm{m} / \mathrm{Br} \gamma$ flux ratio map (middle panel of Fig. 5) shows values smaller than 0.6 at all locations of the CNSFRs ring, indicating that the observed $\mathrm{H}_{2}$ emission is produced by fluorescent excitation due to absorptions of UV photons emitted by young stars.

\subsection{A nuclear Starburst in Mrk 42}

Using the flux values presented in Table 1 for the nucleus of Mrk 42 we obtain very low $\mathrm{H}_{2} \lambda 2.12 \mu \mathrm{m} / \mathrm{Br} \gamma$ and $[\mathrm{Fe}$ II $] \lambda 1.25 \mu \mathrm{m} / \mathrm{Pa} \beta$ values. Both ratios show values smaller than $\sim 0.1$, which are not consistent with those usually observed for Seyfert nuclei (larger than 0.6 for both ratios, Rodríguez-Ardila et al. 2004).

By fitting the Sloan Digital Sky Survey (SDSS; Albareti et al. 2017; Blanton et al. 2017) spectrum of Mrk 42, we obtain $[\mathrm{N}$ II] $\lambda 6583 / \mathrm{H} \alpha \approx 0.2$ and $[\mathrm{O}$ III] $\lambda 5007 / \mathrm{H} \beta \approx 3$, which put the nucleus of Mrk 42 in the Starburst region of the BPT diagram (Baldwin, Phillips \& Terlevich 1981), consistent near-IR line ratios, although it should be noticed that the SDSS spectrum is obtained for an aperture of 3 arcsec diameter, which includes the CNSFRs ring. However, it is well known that this galaxy presents a type 1 AGN, as broad recombination lines are detected. The low-emission-line ratios detected at optical and near-IR can be explained if, besides the central AGN, Mrk 42 presents also strong nuclear Starburst activity, which dominates the gas excitation. Similar nuclear Starbursts are seen in other galaxies with CNSFRs rings, as for NGC 4303 (Colina \& Arribas 1999; Colina et al. 2002; Riffel et al. 2016).

\subsection{Gas kinematics}

The velocity fields presented in Fig. 7 clearly show a rotation component. In order to obtain the kinematical and geometrical parameters, we have fitted the observed $\mathrm{Pa} \beta$ velocities by a rotating disc model, assuming circular orbits in the plane of the galaxy, using the following equation (Bertola et al. 1991):

$$
\begin{aligned}
& V_{\mathrm{r}}(R, \Psi) \\
& \quad=V_{\mathrm{s}}+\frac{A R \cos \left(\Psi-\Psi_{0}\right) \sin (i) \cos ^{p} \theta}{R^{2}\left[\sin ^{2}\left(\Psi-\Psi_{0}\right)+\cos ^{2}(i) \cos ^{2}\left(\Psi-\Psi_{0}\right)\right]+\mathrm{c}_{0}^{2} \cos ^{2}(i)^{p / 2}},
\end{aligned}
$$

where $V_{\mathrm{r}}$ is the circular velocity and $R$ and $\Psi$ are the coordinates of each pixel in the plane of the sky, $V_{\mathrm{s}}$ is the systemic velocity, $A$ is the amplitude of the rotation curve in the plane of the galaxy, $\Psi_{0}$ is the position angle of the line of nodes, $c_{0}$ is a concentration parameter, defined as the radius where the rotation curve reaches 70 percent of the velocity amplitude, $i$ is the disc inclination relative to the plane of the sky and $p$ is a model fitting parameter. The parameter $p$ measures the slope of the rotation curve where it flattens, in the outer region of the galaxy and it is limited between $1 \leq p \leq 3 / 2$. For $p=1$ the rotation curve at large radii is asymptotically flat while for $p=3 / 2$ the system has a finite mass.

We fitted the $\mathrm{Pa} \beta$ velocity field by the equation above using the MPFITFUN routine (Markwardt et al. 2009) to perform a non-linear 

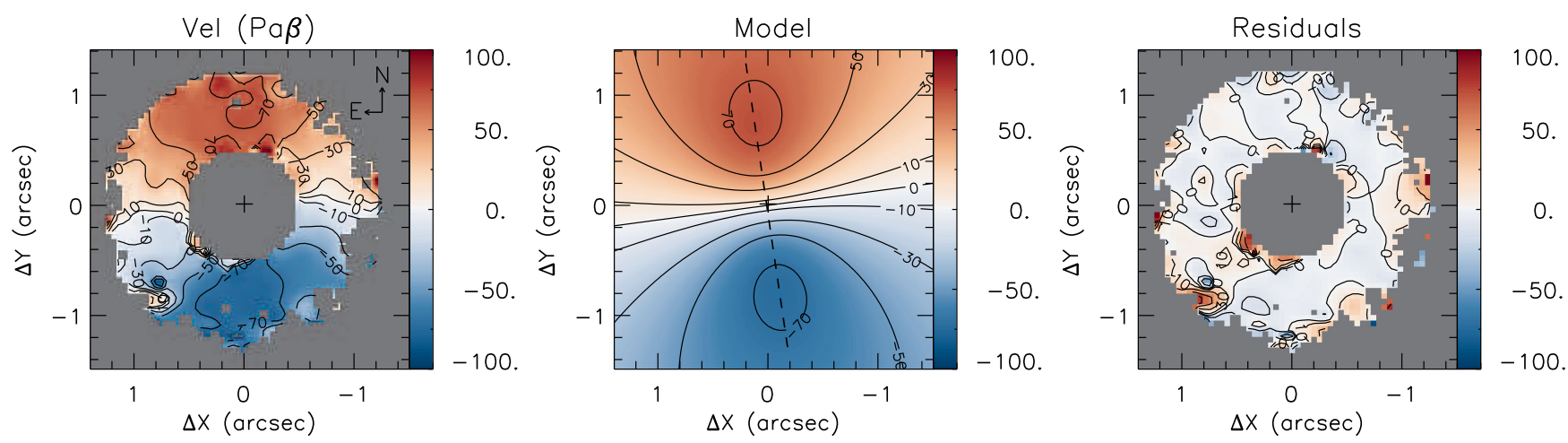

Figure 8. Left-hand panel: $\mathrm{Pa} \beta$ velocity field; middle: rotating disc model and right: residual map, obtained as the difference of the observed velocities and the model. The colour bars show the velocity in units of $\mathrm{km} \mathrm{s}^{-1}$ and the central cross indicates the position of the nucleus. The external grey regions represent masked locations where the signal-to-noise ratio of $\mathrm{Pa} \beta$ was not high enough to allow a good fit of its profile, while the grey region at $r<0.45$ arcsec represents masked locations, where gas outflows are suggested. The central cross marks the position of the nucleus.

least-squares fit, where initial guesses are given for each parameter and the routine returns their values for the best-fitted model. The $\mathrm{Pa} \beta$ velocity field was chosen because the $\mathrm{Pa} \beta$ emission line presents the highest signal-to-noise ratio among the observed emission lines. During the fit, we excluded regions at $r<0.45 \operatorname{arcsec}$ from the nucleus, where an additional kinematic component is observed. During the fit, the position of the kinematical centre was kept fixed at the nucleus, defined as the location of the peak of the continuum emission.

The resulting kinematical model is shown in the middle panel of Fig. 8, while the observed $\mathrm{Pa} \beta$ velocity field is shown in the left-hand panel. Grey locations represent masked positions, where we were not able to obtain good velocity measurements (distances larger than 1 arcsec from the nucleus) and the nuclear region, where outflows from the central AGN seem to co-exist with a disc component. The residual map, obtained as the difference between the observed velocities and the model is presented in the right-hand panel. The residual map presents values very close to zero at all locations (smaller than $10 \mathrm{~km} \mathrm{~s}^{-1}$ ), showing that the velocity field is well represented by the model and thus, dominated by regular rotation.

The resulting parameters for the best-fitting model are: $i=$ $20.2^{\circ} \pm 2.0^{\circ}, V_{\mathrm{s}}=7391 \pm 11 \mathrm{~km} \mathrm{~s}^{-1}$, corrected to the heliocentric reference frame, $A=259 \pm 24 \mathrm{~km} \mathrm{~s}^{-1}, \Psi_{0}=8^{\circ} \pm 1^{\circ}, c_{0}=$ $0.56 \pm 0.05 \operatorname{arcsec}$ and $p=1.5$, which was limited between 1 and 1.5. The disc inclination is slightly larger than that of the largescale disc, obtained assuming $i=a \cos b / a$, where $a$ and $b$ are the semimajor and semiminor axis of the galaxy and the value of the systemic velocity $\left(V_{\mathrm{s}}=7385 \mathrm{~km} \mathrm{~s}^{-1}\right)$ is in a good agreement with that of Falco et al. (1999). The orientation of the line of nodes is similar to that of the large-scale disc, quoted in the Hyperleda data base $\left(\Psi_{0}=-12^{\circ}\right.$, Makarov et al. 2014).

Within the inner $r<0.45$ arcsec radius, the $\mathrm{Pa} \beta$ emission line seems to present two narrow kinematic components: the blueshifted component is seen with velocity of $V_{\text {blue }} \approx-300 \mathrm{~km} \mathrm{~s}^{-1}$, relative to the systemic velocity, while the redshifted component is seen with velocities very close to the nuclear velocity. The blueshifted component is only marginally detected and thus, we were not able to properly fit it. A possible interpretation of these two components is that the blueshifted one is originated from outflowing gas from the nucleus excited by the central AGN, while the redshifted one may be originated from the nuclear Starburst located at the disc of the galaxy.

\subsection{Stellar populations and CNSFRs}

We used the integrated emission-line fluxes of Table 1 to calculate the following properties: the mass of ionized $M_{\mathrm{H} \text { II }}$ gas; rate of ionizing photons $Q\left[\mathrm{H}^{+}\right]$; the SFR and the mass of hot molecular $\mathrm{M}_{\mathrm{H}_{2}}$.

The mass of ionized gas can be estimated by (e.g. Riffel et al. 2016)

$$
\left(\frac{M_{\mathrm{H} \text { І }}}{M_{\odot}}\right)=3 \times 10^{19}\left(\frac{F_{\mathrm{Br} \gamma}}{\mathrm{erg} \mathrm{cm}^{-2} \mathrm{~s}^{-1}}\right)\left(\frac{D}{\mathrm{Mpc}}\right)^{2}\left(\frac{N_{\mathrm{e}}}{\mathrm{cm}^{-3}}\right)^{-1},
$$

where $F_{\mathrm{Br} \gamma}$ is the $\mathrm{Br} \gamma$ flux, $D$ is the distance to the galaxy, and $N_{\mathrm{e}}$ is the electron density, under the assumption of case $\mathrm{B}$ recombination at an electron temperature of $10^{4} \mathrm{~K}$ (Osterbrock \& Ferland 2006). The mass of hot molecular gas can be estimated by (Scoville et al. 1982; Riffel et al. 2008)

$$
\left(\frac{M_{\mathrm{H}_{2}}}{M_{\odot}}\right)=5.0776 \times 10^{13}\left(\frac{F_{\mathrm{H}_{2} \lambda 2.1218}}{\mathrm{erg} \mathrm{cm}^{-2} \mathrm{~s}^{-1}}\right)\left(\frac{D}{\mathrm{Mpc}}\right)^{2},
$$

where $F_{\mathrm{H}_{2} \lambda 2.1218}$ is the flux of the $\mathrm{H}_{2} \lambda 2.1218$ emission line and we assume local thermal equilibrium and an excitation temperature of $2000 \mathrm{~K}$ for the $\mathrm{H}_{2}$.

The emission rate of ionizing photons is obtained following Riffel et al. (2009):

$$
\left(\frac{Q\left[\mathrm{H}^{+}\right]}{\mathrm{s}^{-1}}\right)=7.47 \times 10^{13}\left(\frac{L_{\mathrm{Br} \gamma}}{\mathrm{erg} \mathrm{s}^{-1}}\right),
$$

where $L_{\mathrm{Br} \gamma}$ is the $\mathrm{Br} \gamma$ luminosity and the SFR is obtained by

$$
\left(\frac{\mathrm{SFR}}{\mathrm{M}_{\odot} \mathrm{yr}^{-1}}\right)=8.2 \times 10^{-40}\left(\frac{L_{\mathrm{Br} \gamma}}{\mathrm{erg} \mathrm{s}^{-1}}\right),
$$

following Kennicutt (1998). The equations above for SFR and $Q\left[\mathrm{H}^{+}\right]$are derived under the assumption of continuous star formation, and should be considered just proxies of these parameters.

In order to estimate the mass of ionized gas and the resulting physical properties of each CNSFR of Mrk 42 that are shown in Table 3, we have assumed the electron density value of $N_{\mathrm{e}}=300$ $\mathrm{cm}^{-3}$, which is the mean value of the electron density for a sample of CNSFRs derived from the $\left[\mathrm{S}_{\mathrm{II}}\right] \lambda 6717 / \lambda 6731$ intensity ratio by Díaz et al. (2007) and Dors et al. (2008).

The derived physical parameters for the CNSFRs of Mrk 42 can be compared with previously published values. Dors et al. (2008) 
Table 3. Physical parameters of the CNSFRs in Mrk 42 from integrated emission-line fluxes shown in Table 1. The location of each region is indicated in Fig. 4. $Q\left[\mathrm{H}^{+}\right]$is the ionizing photons rate, $\mathrm{SFR}$ the star formation rate, $M_{\mathrm{H}}$ iा is the mass of ionized gas, and $\mathrm{M}_{\mathrm{H}_{2}}$ the mass of hot molecular gas.

\begin{tabular}{lccrr}
\hline Region & $\log Q\left[\mathrm{H}^{+}\right]\left(\mathrm{s}^{-1}\right)$ & $\mathrm{SFR}\left(10^{-2} \mathrm{M}_{\odot} \mathrm{yr}^{-1}\right)$ & $\mathrm{M}_{\mathrm{H} \text { II }}\left(10^{4} \mathrm{M}_{\odot}\right)$ & $\mathrm{M}_{\mathrm{H}_{2}}\left(\mathrm{M}_{\odot}\right)$ \\
\hline A & $52.3 \pm 0.06$ & $21.5 \pm 3.30$ & $21.9 \pm 3.36$ & $43.0 \pm 9.20$ \\
B & $51.8 \pm 0.06$ & $7.4 \pm 1.20$ & $7.6 \pm 1.23$ & $23.6 \pm 6.65$ \\
C & $51.8 \pm 0.06$ & $7.4 \pm 1.17$ & $7.5 \pm 1.19$ & $23.6 \pm 5.30$ \\
D & $51.8 \pm 0.07$ & $8.6 \pm 1.43$ & $7.7 \pm 1.46$ & $23.0 \pm 4.72$ \\
E & $51.9 \pm 0.05$ & $7.8 \pm 0.93$ & $8.4 \pm 0.95$ & $17.9 \pm 3.67$ \\
F & $51.8 \pm 0.05$ & $6.9 \pm 0.89$ & $7.9 \pm 0.96$ & $38.5 \pm 7.86$ \\
G & $51.8 \pm 0.05$ & $6.4 \pm 1.27$ & $6.0 \pm 0.91$ & $25.7 \pm 4.25$ \\
H & $51.8 \pm 0.08$ & $138.7 \pm 12.89$ & $141.43 \pm 13.13$ & $29.6 \pm 4.13$ \\
Ring & $53.1 \pm 0.04$ & & $624.3 \pm 87.19$ \\
\hline
\end{tabular}

used optical IFS to study the CNSFRs of the Seyfert 2 galaxies NGC 1097 and NGC 6951 and found SFR in the range 0.002$0.14 \mathrm{M}_{\odot} \mathrm{yr}^{-1}$ for the eight star-forming regions studied. Shi, Gu \& Peng (2006) used the observations from the SDSS to study a sample of 385 objects classified as star-forming galaxies and found an average value for the SFR of $0.14 \mathrm{M}_{\odot} \mathrm{yr}^{-1}$. Falcón-Barroso et al. (2014) used SINFONI data to study the star formation in the inner $700 \mathrm{pc}$ of the active spiral galaxy NGC 613 and obtained SFR $\sim 10^{-2}$ to $10^{-1} \mathrm{M}_{\odot} \mathrm{yr}^{-1}$ for eight CNSFRs. Riffel et al. (2016) found SFR of (0.4-2.0) $\times 10^{-2} \mathrm{M}_{\odot} \mathrm{yr}^{-1}$ for the CNSFRs of NGC 4303 derived from SINFONI observations. Thus, the values of SFR derived for the ring of Mrk $42\left(0.07-0.2 \mathrm{M}_{\odot} \mathrm{yr}^{-1}\right)$ are within the range of values observed for other galaxies with similar nuclear rings.

The emission rates of ionizing photons for the CNSFRs of Mrk 42 are in the range $\log (Q)=51.8-52.3$ photons $\mathrm{s}^{-1}$, in agreement with values already obtained for CNSFRs in other galaxies (e.g. Wold \& Galliano 2006; Galliano \& Alloin 2008; Riffel et al. 2009, 2016). Such rates correspond to more than hundred O3 stars in each CNSFR (Osterbrock \& Ferland 2006), but these values should be considered only as a proxy as a continuous star formation regime was assumed and the most probable scenario may be that of instantaneous star formation.

Finally, the masses of ionized and hot molecular gas observed for Mrk 42 are similar to that observed for other CNSFRs (e.g. Riffel et al. 2016, 2009). The average ratio between the ionized and hot molecular gas masses is $\left\langle M_{\mathrm{H}} / M_{\mathrm{H}_{2}}\right\rangle \sim 3312$, being similar to that observed for the CNSFRs of NGC 4303 (2030, Riffel et al. 2016), and also in the range of values obtained for the inner kiloparsec of nearby Seyfert galaxies, of 200-8000 (e.g. Schönell et al. 2017; Riffel et al. 2018).

The total mass of ionized gas in the ring (derived by measuring the fluxes of $\mathrm{Br} \gamma$ and $\mathrm{H}_{2} \lambda 2.12 \mu \mathrm{m}$ from an integrated spectrum comprising distances between 0.45 and 1.3 arcsec from the nucleus is 1.9 times larger than the sum of $M_{\mathrm{H} \text { II }}$ of all CNSFRs. The total mass of hot molecular gas in the ring is 2.8 times larger than that of the CNSFRs, indicating that a large amount of gas is observed outside the CNSFRs.

We can also use the spectra shown in Fig. 2 to better investigate the stellar content of the CNSFRs. These spectra show strong absorption features at 1.17-1.20 $\mu \mathrm{m}$, which may be molecular absorptions from stars in the thermally pulsing asymptotic giant branch (TP-AGB) phase, as suggested by simple stellar populations models (Maraston 2005) and detected in the nuclear spectra of nearby AGNs (e.g. VO and CN features, Riffel et al. 2007, 2015). In addition, these features are clearly present in the spectra of stars in the TP-AGB phase (Lançon \& Mouhcine 2002; Maraston 2005; Rayner, Cushing \& Vacca 2009). By comparing the spectra of the
CNSFRs 'E', 'F', and ' $G$ ', with the stellar spectra shown by Lançon $\&$ Mouhcine (2002), one can see that similar features are also seen in the spectra of oxygen (e.g. from $\mathrm{TiO}$ and $\mathrm{VO}$ ) and carbon (e.g. CN features) rich stars in the upper AGB phase. Martins et al. (2013a) also reported the detection of signatures of stellar population dominated by the TP-AGB (as $\mathrm{CN}$, TiO, and $\mathrm{ZrO}$ absorptions) in near-IR spectra of $\mathrm{H}$ II galaxies, which show a similar absorption features at $\sim 1.19 \mu \mathrm{m}$ as seen in the CNSFRs of Mrk 42. However, stellar population synthesis performed for star-forming galaxies by Martins et al. (2013b) do not find a clear correlation between the near-IR (e.g. CN) indexes and the presence of intermediate-age stellar populations, but this is attributed by these authors as due to observational limitations. Thus, the detection of these molecular features in the CNSFRs ' $E$ ', ' $F$ ', and ' $G$ ' suggests that these regions are older than the other CNSFRs of Mrk 42 and already present some evolved stars.

\subsection{How does the star formation proceed in the CNSFRs ring?}

Gas inflows towards the central region of galaxies provide a gas reservoir, which can trigger (circum-)nuclear star formation and a central AGN. The way the material flows towards the central region may be through bars, spiral arms, or interactions with neighbour galaxies. Inflows of gas associated with nuclear dust spirals and bars have been observed for nearby active galaxies at scales of a few hundred parsecs, mostly using optical and near-IR IFS (e.g. Fathi et al. 2006; Storchi-Bergmann et al. 2007; Riffel et al. 2008; Müller Sánchez et al. 2009; van de Ven \& Fathi 2010; Riffel et al. 2013a; Schnorr-Müller al. 2014a; Schnorr-Müller et al. 2017), but the presence of nuclear dust structures is not a sufficient condition to produce gas streaming (Brum et al. 2017).

Two possible scenarios have been proposed for the origin of the CNSFRs at scales of few hundred parsecs: the popcorn and pearls on a string scenarios (Böker et al. 2008) that show distinct distributions in age of the stellar clusters. In the popcorn scenario, the gas is accumulated in the circumnuclear region resulting in a ring of higher density material, which allows a simultaneous star formation in the whole ring or in individual hotsposts, collapsing at different times within the ring. Thus, for this scenario there is no relation between the age of the recent formed stellar clusters and their locations within the ring.

In the pearls on a string scenario, a short-lived, quasiinstantaneous burst of star formation is induced and located in a certain region of the ring. The locations of these ODRs (where the gas density is high enough to trigger the star formation) depend on details of the gravitational potential, but they are frequently found at locations where the gas reaches the central region, as for example, 


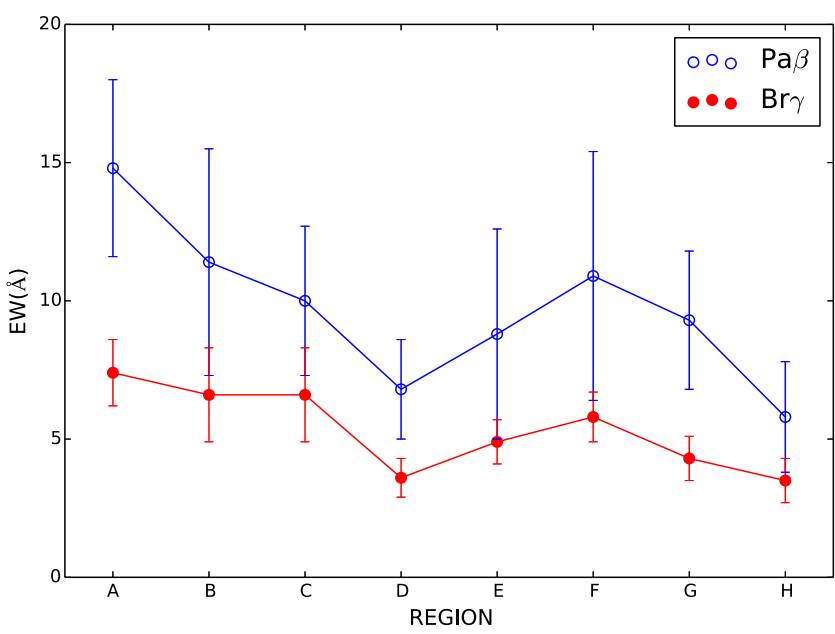

Figure 9. Sequence of values for the $\mathrm{EqW}$ of $\mathrm{Pa} \beta$ (open blue circles) and $\operatorname{Br} \gamma$ (filled red circles) emission lines for the CNSFRs labelled from 'A' to ' $\mathrm{H}$ ' in Fig. 4.

at the tips of bars. Stellar clusters are formed in these ODRs, which follow orbits within the ring, resulting in several stellar clusters with a sequence of ages, and are called 'pearls on a string'. Thus, in this scenario, two segments of stellar clusters, on opposite sides of the ring, are expected in which the youngest clusters have their locations near the ODRs, becoming older when approaching the opposite ODR, i.e. there is a correlation between the stellar cluster age and the ring localization.

In order to distinguish between the popcorn and pearls on a string scenarios for the origin of the ring of CNSFRs in Mrk 42, we constructed Fig. 9 that shows the sequence of $\mathrm{EqW}$ values for $\mathrm{Pa} \beta$ (open blue circles) and $\operatorname{Br} \gamma$ (filled red circles) emission lines for the regions labelled from 'A' to ' $\mathrm{H}$ '. The EqW values were measured from the integrated spectra of Fig. 2 and are shown in Table 2. The EqW values of $\mathrm{H}$ recombination lines can be used to determine the age of the star formation regions, with higher values observed for younger regions and lower values for older regions, under the assumption that all regions are intrinsically equal in stellar mass and the mass in the burst is the same (Dottori 1981; Copetti, Pastoriza \& Dottori 1986). By comparing $\mathrm{Br} \gamma \mathrm{EqW}$ values observed for the CNSFRs of Mrk 42 with the predicted values by evolutionary photoionization models (Dors et al. 2008; Riffel et al. 2009), we conclude that all regions have ages between 5 and $6 \mathrm{Myr}$, considering the correction due to the contribution to the bulge stars to the underlying continuum in the derived $\mathrm{EqW}$ values, as discussed in Section 3.1. The highest EqW values are observed for region 'A', decreasing to a minimum value for region ' $D$ '. A second gradient is seen from region ' $F$ ' to ' $\mathrm{H}$ '. As the $\mathrm{EqW}$ of the $\mathrm{H}$ recombination lines can be associated with the age of the stars, these gradients can be interpreted as a signature of two age sequences, one from region 'A' to 'D' and another from region ' $F$ ' to ' $\mathrm{H}$ ', favouring thus the pearls on a string scenario if the gas reaches the centre along the major axis of the galaxy (close to the orientation of the bar - see Fig. 4). On the other hand, the spectra for the regions ' $E$ ', ' $F$ ', and ' $G$ ' clearly show the presence of the molecular absorption bands at $1.17-1.20 \mu \mathrm{m}$. These absorptions (TiO, $\mathrm{CN}$, or $\mathrm{VO}$ ) are originated in more evolved stars that are in the TP-AGB phase (e.g. Maraston 2005), indicating that these regions are older than the remaining and suggests no clear sequence in age for these CNSFRs and thus not supporting the above sequence of ages for these CNSFRs.

\subsection{Mass of the SMBH}

The mass of the SMBH can be obtained directly from the $\mathrm{Pa} \beta$ width and luminosity using the following equation (Kim, Im \& Kim 2010; Kim et al. 2015; Woo et al. 2015):

$\frac{M_{\mathrm{BH}}}{M_{\odot}}=10^{7.04 \pm 0.02}\left(\frac{L_{\mathrm{Pa} \beta}}{10^{42} \mathrm{erg} \mathrm{s}^{-1}}\right)^{0.48 \pm 0.03}\left(\frac{\mathrm{FWHM}_{\mathrm{Pa} \beta}}{10^{3} \mathrm{~km} \mathrm{~s}^{-1}}\right)^{2}$,

where $L_{\mathrm{Pa} \beta}$ is the luminosity and $\mathrm{FWHM}_{\mathrm{Pa} \beta}$ corresponds to width of the broad $\mathrm{Pa} \beta$ component. Assuming $L_{\mathrm{Pa} \beta}=(9.0 \pm$ $0.3) \times 10^{39} \mathrm{erg} \mathrm{s}^{-1}$ and $\mathrm{FWHM}_{\mathrm{Pa} \beta}=1480 \pm 40 \mathrm{~km} \mathrm{~s}^{-1}$, measured from the $\mathrm{Pa} \beta$ profile within a circular aperture of 0.25 arcsec radius, we obtain $M_{\mathrm{BH}}=2.5_{-0.6}^{+0.7} \times 10^{6} \mathrm{M}_{\odot}$.

The mass of the SMBH of Mrk 42 derived here is in good agreement with values obtained using scaling relations based on optical observations and the X-ray excess variance, which are in the range $(0.7-18) \times 10^{6} \mathrm{M}_{\odot}$ (Wang \& Lu 2001; Bian \& Zhao 2003; Nikolajuk et al. 2009).

We notice that the width of the $\mathrm{Pa} \beta$ broad emission line is larger by a factor of 1.7-2.2 than those quoted in the literature for the $\mathrm{H} \beta$ broad component (Goodrich 1989; Wang \& Lu 2001; Bian \& Zhao 2003). However, it should be noted that the values of FWHM for $\mathrm{H} \beta$ available in the literature are based on more than $20 \mathrm{yr}$ old low resolution spectra. Using the SDSS (Albareti et al. 2017; Blanton et al. 2017) spectrum of Mrk 42, we note that the broad $\mathrm{H} \beta$ component is just marginally detected, but its width is consistent with our measurement for $\mathrm{Pa} \beta$. The $\mathrm{H} \alpha$ broad component of Mrk 42 is clearly detected in the SDSS spectrum and has an FWHM $\approx$ $1400 \mathrm{~km} \mathrm{~s}^{-1}$, being similar to that derived for $\mathrm{Pa} \beta$.

\section{CONCLUSIONS}

We used $J, H$ and $K$ bands Gemini NIFS observations to map the flux distributions and kinematics in the near-IR emission lines of the inner $1.5 \times 1.5 \mathrm{kpc}^{2}$ of Mrk 42 at a spatial resolution of $60 \mathrm{pc}$ and velocity resolution of $\sim 40 \mathrm{~km} \mathrm{~s}^{-1}$. The main conclusions of this work are

(i) The emission-line flux distributions and EqW maps clearly show the ring of CNSFRs, previously detected in HST image at $\sim 300$ pc from the nucleus. We detected eight CNSFRs (labelled from ' $\mathrm{A}$ ' to ' $\mathrm{H}$ ') in the ring and by comparing the observed EqW values of the $\operatorname{Br} \gamma$ emission line with those predicted by photoionization models, we conclude that their age is between 5 and $6 \mathrm{Myr}$. These regions present SFRs in the range 0.06-0.21 $\mathrm{M}_{\odot} \mathrm{yr}^{-1}$, ionizing photons rate of $\log (Q)=51.8-52.3$ photons $^{-1}$, masses of ionized gas in the range $(6.5-22) \times 10^{4} \mathrm{M}_{\odot}$, and masses of hot molecular gas between 18 and $43 \mathrm{M}_{\odot}$. These masses represent $\approx 53$ per cent and 36 per cent of the total masses in the ring, respectively.

(ii) In addition to a central AGN, line-intensity ratios indicate that Mrk 42 harbours a nuclear Starburst.

(iii) The spectra of the regions ' $E$ ', ' $F$ ', and ' $G$ ' show molecular absorption bands at $1.17-1.20 \mu \mathrm{m}$, possibly originated from evolved stars at the TP-AGB phase.

(iv) The near-IR emission lines of $[\mathrm{Fe} \mathrm{II}]$ and $\mathrm{H}_{2}$ from the starforming regions have as main ionizing source young stars with 5-6 Myr of age.

(v) The gas velocities at locations beyond the inner 0.45 arcsec $(\sim 220 \mathrm{pc})$ are dominated by rotation, being well reproduced by a disc model assuming circular orbits in the plane of the galaxy. At the nucleus, the gas kinematics suggests two components: one due to the disc of the galaxy, with $V_{\mathrm{LOS}}$ similar to the systemic velocity of 
the galaxy $\left(7391 \pm 11 \mathrm{~km} \mathrm{~s}^{-1}\right)$ and another blueshifted by $300-500$ $\mathrm{km} \mathrm{s}^{-1}$, attributed to a nuclear outflow.

(vi) The EqW values of the $\mathrm{Br} \gamma$ emission line suggest two age sequences, one from region ' $A$ ' to ' $D$ ' and another from region ' $F$ ' to ' $G$ '. These age sequences favour the 'pearls on a string' scenario for the origin of the CNSFRs in Mrk 42, although the presence of signatures of TP-AGB stars in some of the regions undermines this interpretation.

(vii) The FWHM of the broad component of $\mathrm{Pa} \beta$ emission line is $\sim 1480 \mathrm{~km} \mathrm{~s}^{-1}$, being larger than the values previously observed for $\mathrm{H} \beta$. Using this value we obtain a mass of $M_{\mathrm{BH}}=2.5_{-0.6}^{+0.7} \times 10^{6}$ $\mathrm{M}_{\odot}$ for the central supermassive black hole.

\section{ACKNOWLEDGEMENTS}

We thank the anonymous referee for valuable suggestions which helped to improve the paper. This work is based on observations obtained at the Gemini Observatory, which is operated by the Association of Universities for Research in Astronomy, Inc., under a cooperative agreement with the NSF on behalf of the Gemini partnership: the National Science Foundation (United States), the Science and Technology Facilities Council (United Kingdom), the National Research Council (Canada), CONICYT (Chile), the Australian Research Council (Australia), Ministério da Ciência e Tecnologia (Brazil), and south-eastCYT (Argentina). This research has made use of the NASA/IPAC Extragalactic Database (NED) which is operated by the Jet Propulsion Laboratory, California Institute of Technology, under contract with the National Aeronautics and Space Administration. We acknowledge the usage of the HyperLeda data base (http://leda.univ-lyon1.fr). The authors acknowledge support from Conselho Nacional de Desenvolvimento Científico e Tecnológico (CNPq) and Fundaç cão de Amparo à Pesquisa do Estado do Rio Grande do Sul (FAPERGS). OLD is grateful to Fundaç cão de Amparo à Pesquisa do Estado de São Paulo (FAPESP) (2016/04728-7) and CNPQ (306744/2014-7).

\section{REFERENCES}

Albareti F. D et al., 2017, ApJS, 233, 25

Baldwin J. A., Phillips M. M., Terlevich R., 1981, PASP, 93, 5

Bertola F., Bettoni D., Danziger J., Sadler E., Sparke L., de Zeeuw T., 1991, ApJ, 373, 369

Bian W., Zhao Y., 2003, MNRAS, 343, 164

Black J. H., van Dishoeck E. F., 1987, ApJ, 322, 412

Blanton M. R. et al., 2017, AJ, 154, 28

Böker T., Falcón-Barroso J., Schinnerer E., Knapen J. H., Ryder S., 2008, AJ, 135, 479

Brum C., Riffel R. A., Storchi-Bergmann T., Robinson A., Schnorr-Muller A., Lena D., 2017, MNRAS, 469, 3405

Cardelli J. A., Clayton G. C., Mathis J. S., 1989, ApJ, 345, 245

Colina L., Arribas S., 1999, ApJ, 514, 637

Colina L., Gonzalez Delgado R., Mas-Hesse J. M., Leitherer C., 2002, ApJ, 579,545

Colina L. et al., 2015, A\&A, 578, 48

Copetti M. V. F., Pastoriza M. G., Dottori H. A., 1986, A\&A, 156, 111

Davies R. I. I., Sternberg A., Lehnert M. D., Tacconi- Garman L. E., 2005, ApJ, 633, 105

Davies R. I., Müller Sánchez F., Genzel R., Tacconi L. J., Hicks E. K. S., Friedrich S., Sternberg A., 2007, ApJ, 671, 1388

Deo R. P., Crenshaw D. M., Kraemer S. B., 2006, AJ, 132, 321

Díaz Á. I., Terlevich E., Castellanos M., Hägele G. F., 2007, MNRAS, 382, 251

Diniz M. R., Riffel R. A., Storchi-Bergmann T., Winge C., 2015, MNRAS, 453,1727
Dopita M. A., Sutherland R. S., 1996, ApJS, 102, 161

Dors O. L., Storchi-Bergmann T., Jr, Riffel R. A., Schimdt A. A., 2008, A\&A, 482, 59

Dors O. L., Jr et al., 2012, MNRAS, 422, 252

Dottori H. A., 1981, Ap\&SS, 80, 267

Elmegreen B. G., 1994, ApJ, 425, L73

Falco E. E. et al., 1999, PASP, 111, 438

Falcón-Barroso J., Ramos Almeida C., Böker T., Schinnerer E., Knapen J. H., Lançon A., Ryder S., 2014, MNRAS, 438, 329

Fathi K., Storchi-Bergmann T., Riffel R. A., Winge C., Axon D. J., Robinson A., Capetti A., Marconi A., 2006, ApJl, 641, L25

Ferrarese L., Merritt D., 2000, ApJ, 539, L9

Forbes D. A., Ward M. J., 1993, ApJ, 416, 150

Galliano E., Alloin D., 2008, A\&A, 487, 519

Gebhardt K. et al., 2000, ApJ, 539, L13

Goodrich R. W., 1989, ApJ 342, 224

Hollenbach D., McKee C. F., 1989, ApJ, 342, 306

Hunt L. K., Malkan M. A., 1999, ApJ, 516, 660

Hunt L. K., Malkan M. A., Rush B., Bicay M. D., Nelson B. O., Stanga R. M., Webb W., 1999, ApJS, 125, 349

Jogee S., Scoville N., Kenney J. D. P., 2005, ApJ, 630, 837

Kennicutt R. C., Jr, 1998, ARA\&A, 36, 189

Kennicutt R. C., Keel W., Blaha C. A., 1989, ApJ, 97, 1022

Kim D., Im M., Kim M., 2010, ApJ, 724, 386

Kim D., Im M., Glikman E., Woo J.-H., Urrutia T., 2015, ApJ, 812, 66

Knapen J. H., 2005, A\&A, 429, 141

Kotilainen J. K., Reunanen J., Laine S., Ryder S. D., 2000, A\&A, 353, 834

Lamperti I. et al., 2017, MNRAS, 467, 540

Lançon A., Mouhcine M., 2002, A\&A, 393, 167

Larkin J. E., Armus L., Knop R. A., Soifer B. T., Matthews K., 1998, ApJS, 114,59

Makarov D., Prugniel P., Terekhova N., Courtois H., Vauglin I., 2014, A\&A, 570,13

Malkan M. A., Gorjian V., Tam R., 1998, ApJS, 117, 25

Maloney P. R., Hollenbach D. J., Tielens A. G. G. M., 1996, ApJ, 466, 561

Maraston C., 2005, MNRAS, 362, 799

Markwardt C. B., 2009, in Bohlender D. A., Durand D., Dowler P., eds, ASP Conf. Ser. Vol. 411, Astronomical Data Analysis Software and Systems XVIII. Astron. Soc. Pac., San Francisco, p. 251

Martins L. P., Rodríguez-Ardila A., Diniz S., Gruenwald R., de Souza R., 2013a, MNRAS, 431, 1823

Martins L. P., Rodríguez-Ardila A., Diniz S., Riffel R., de Souza R., 2013b, MNRAS, 435, 2861

May D., Steiner J. E., Ricci T. V., Menezes R. B., Andrade I. S., 2016, MNRAS, 457, 949

Mazzuca L. M., Knapen J. H., Veilleux S., Regan M. W., 2008, ApJS, 174, 337

McGregor P. J. et al., 2003, in Iye M., Moorwood A. F. M., eds, Proc. SPIE Conf. Ser. Vol. 4841, Instrument Design and Performance for Optical/Infrared Ground-based Telescopes. SPIE, Bellingham, p. 1581

Morgan W. W., 1958, PASP, 70, 364

Müller Sánchez F., Davies R. I., Genzel R., Tacconi L. J., Eisenhauer F., Hicks E. K. S., Friedrich S., Sternberg A., 2009, ApJ, 691, 749

Muñoz Marín V. M., González Delgado R. M., Schmitt H. R., Cid Fernandes R., Pérez E., Storchi-Bergmann T., Heckman T., Leitherer C., 2007, AJ, 134,648

Nikolajuk M., Czerny B., Gurynowicz P., 2009, MNRAS, 394, 2141

Norman C., Scoville N., 1988, ApJ, 332, 124

Osterbrock D. E., Ferland G. J., 2006, Astrophysics of Gaseous Nebulae and Active Galactic Nuclei, 2nd edn. University Science Books, Mill Valley, CA

Perry J. J., Dyson J. E., 1985, MNRAS, 213, 665

Ramos Almeida C., Pérez García A. M., Acosta-Pulido J. A., 2009, ApJ, 694, 1379

Rayner J. T., Cushing M. C., Vacca W. D., 2009, ApJS, 185, 289

Reunanen J., Kotilainen J. K., Prieto M. A., 2002, MNRAS, 331, 154

Ricci T. V., Steiner J. E., Menezes R. B., 2014, MNRAS, 440, 2419 
Riffel R. A., 2010, Ap\&SS, 327, 239

Riffel R. A., Sorchi-Bergmann T., Winge C., Barbosa F. K. B., 2006, MNRAS, 373, 2

Riffel R., Pastoriza M. G., Rodriguez-Ardila A., Maraston C., 2007, ApJ, 659, 103

Riffel R. A., Storchi-Bergmann T., Winge C., McGregor P. J., Beck T., Schmitt H., 2008, MNRAS, 385, 1129

Riffel R. A., Storchi-Bergmann T., Dors O. L., Winge C., 2009, MNRAS, 393, 783

Riffel R. A., Storchi-Bergmann T., Nagar N. M., 2010, MNRAS, 404, 166

Riffel R., Bonatto C., Cid Fernandes R., Pastoriza M. G., Balbinot E., 2011, MNRAS, 411, 1897

Riffel R., Rodríguez-Ardila A., Aleman I., Brotherton M. S., Pastoriza M. G., Bonatto C., Dors O. L., 2013a, MNRAS, 430, 2002

Riffel R. A., Storchi-Bergmann T., Winge C., 2013b, MNRAS, 430, 2249

Riffel R. et al., 2015, MNRAS, 450, 3069

Riffel R. A. et al., 2016, MNRAS, 461, 4192

Riffel R. A. et al., 2018, MNRAS, 474, 1373

Rodríguez-Ardila A., Pastoriza M. G., Viegas S., Sigut T. A. A., Pradhan A. K., 2004, A\&A, 425, 457

Rodríguez-Ardila A., Riffel R., Pastoriza M. G., 2005, MNRAS, 364, 1041

Sakamoto K., Okumura S. K., Ishizuki S., Scoville N. Z., 1999, ApJ, 525, 691

Sani E., Lutz D., Risaliti G., Netzer H., Gallo L. C., Trakhtenbrot B., Sturm E., Boller T., 2010, MNRAS, 403, 1246

Schnorr-Müller A., Storchi-Bergmann T., Nagar N. M., Lena D., Riffel R. A., Couto G. S., 2014a, MNRAS, 438, 3322

Schnorr-Müller A., Storchi-Bergmann T., Nagar N. M., Robinson A., Ferrari F., 2014b, MNRAS, 437, 1708
Schnorr-Müller A., Storchi-Bergmann T., Ferrari F., Nagar N. M., 2017, MNRAS, 466, 4370

Schönell A. J., Riffel R. A., Storchi- Bergmann T., Riffel R., 2017, MNRAS, 464,1771

Schwarz M. P., 1981, ApJ, 247, 77

Scoville N. Z., Hall D. N. B., Ridgway S. T., Keinmann S. G., 1982, ApJ, 253, 136

Sérsic J. L., Pastoriza M., 1967, PASP, 79, 152

Shi L., Gu Q. S., Peng Z. X., 2006, A\&A, 450, 15

Simões Lopes R. D., Storchi-Bergmann T., de Fátima Saraiva M., Martini P., 2007, ApJ, 655, 718

Simpson C., Forbes D. A., Baker A. C., Ward M. J., 1996, MNRAS, 283, 777

Storchi-Bergmann T., 2008, Rev. Mex. Astron. Astrofis. Conf. Ser., 32, 139

Storchi-Bergmann T., Dors O., Jr, Riffel R. A., Fathi K., Axon D. J., Robinson A., 2007, ApJ, 670, 959

van de Ven G., Fathi K., 2010, ApJ, 723, 767

van der Laan T. P. R. et al., 2015, A\&A, 575, A83

Wang T., Lu Y., 2001, A\&A, 377, 52

Wilson A. S., Helfer T. T., Haniff C. A., Ward M. J., 1991, ApJ, 381, 79

Wold M., Galliano E., 2006, MNRAS, 369, 47

Woo J.-H., Yoon Y., Park S., Park D., Kim S. C., 2015, ApJ, 801, 38

This paper has been typeset from a $\mathrm{TE}_{\mathrm{E}} \mathrm{X} / \mathrm{AT} \mathrm{T} \mathrm{X}$ file prepared by the author. 